\title{
Measurement of leading neutron production in deep-inelastic scattering at HERA
}

The H1 Collaboration

F.D. Aaron ${ }^{6, i}$, C. Alexa ${ }^{6}$, V. Andreev ${ }^{26}$, B. Antunovic ${ }^{12}$, S. Backovic ${ }^{31}$, A. Baghdasaryan ${ }^{39}$, E. Barrelet $^{30}$, W. Bartel $^{12}$, K. Begzsuren ${ }^{36}$, A. Belousov ${ }^{26}$, J.C. Bizot ${ }^{28}$, V. Boudry ${ }^{29}$, I. Bozovic-Jelisavcic ${ }^{2}$, J. Bracinik ${ }^{3}$, G. Brandt ${ }^{12}$, M. Brinkmann ${ }^{13, k}$, V. Brisson ${ }^{28}$, D. Bruncko ${ }^{17}$, A. Bunyatyan ${ }^{14,39}$, G. Buschhorn ${ }^{27}$, L. Bystritskaya ${ }^{25}$, A.J. Campbell ${ }^{12}$, K.B. Cantun Avila ${ }^{23}$, K. Cerny ${ }^{33}$, V. Cerny ${ }^{17, g}$, V. Chekelian ${ }^{27}$, A. Cholewa ${ }^{12}$, J.G. Contreras $^{23}$, J.A. Coughlan ${ }^{7}$, G. Cozzika ${ }^{11}$, J. Cvach ${ }^{32}$, J.B. Dainton ${ }^{19}$, K. Daum ${ }^{38, c}$, M. Deák ${ }^{12}$, B. Delcourt ${ }^{28}$, J. Delvax ${ }^{4,5}$, E.A. De Wolf ${ }^{4,5}$, C. Diaconu ${ }^{22}$, V. Dodonov ${ }^{14}$, A. Dossanov $^{27}$, A. Dubak ${ }^{31, f}$, G. Eckerlin ${ }^{12}$, V. Efremenko ${ }^{25}$, S. Egli ${ }^{37}$, A. Eliseev $^{26}$, E. Elsen ${ }^{12}$, A. Falkiewicz ${ }^{8}$, L. Favart ${ }^{4,5}$, A. Fedotov ${ }^{25}$, R. Felst ${ }^{12}$, J. Feltesse ${ }^{11, h}$, J. Ferencei ${ }^{17}$, D.-J. Fischer ${ }^{12}$, M. Fleischer ${ }^{12}$, A. Fomenko ${ }^{26}$, E. Gabathuler ${ }^{19}$, J. Gayler ${ }^{12}$, S. Ghazaryan ${ }^{12}$, A. Glazov ${ }^{12}$, I. Glushkov ${ }^{40}$, L. Goerlich ${ }^{8}$, N. Gogitidze ${ }^{26}$, M. Gouzevitch ${ }^{12}$, C. Grab ${ }^{41}$, T. Greenshaw ${ }^{19}$, B.R. Grell ${ }^{12}$, G. Grindhammer ${ }^{27}$, S. Habib ${ }^{13}$, D. Haidt ${ }^{12}$, C. Helebrant ${ }^{12}$, R.C.W. Henderson ${ }^{18}$, E. Hennekemper ${ }^{16}$, H. Henschel ${ }^{40}$, M. Herbst ${ }^{16}$, G. Herrera ${ }^{24}$, M. Hildebrandt ${ }^{37}$, K.H. Hiller ${ }^{40}$, D. Hoffmann ${ }^{22}$, R. Horisberger $^{37}$, T. Hreus ${ }^{4,5, d}$, M. Jacquet ${ }^{28}$, X. Janssen ${ }^{4,5}$, L. Jönsson ${ }^{21}$, A.W. Jung ${ }^{16}$, H. Jung ${ }^{12}$, M. Kapichine ${ }^{10}$, J. Katzy ${ }^{12}$, I.R. Kenyon ${ }^{3}$, C. Kiesling ${ }^{27}$, M. Klein ${ }^{19}$, C. Kleinwort ${ }^{12}$, T. Kluge ${ }^{19}$, A. Knutsson ${ }^{12}$, R. Kogler ${ }^{27}$, P. Kostka ${ }^{40}$, M. Kraemer ${ }^{12}$, K. Krastev ${ }^{12}$, J. Kretzschmar ${ }^{19}$, A. Kropivnitskaya ${ }^{25}$, K. Krüger ${ }^{16, a}$, K. Kutak ${ }^{12}$, M.P.J. Landon ${ }^{20}$, W. Lange ${ }^{40}$, G. Laštovička-Medin ${ }^{31}$, P. Laycock ${ }^{19}$, A. Lebedev ${ }^{26}$, V. Lendermann ${ }^{16}$, S. Levonian $^{12}$, G. Li $^{28}$, K. Lipka ${ }^{12, k}$, A. Liptaj ${ }^{27}$, B. List ${ }^{13}$, J. List ${ }^{12}$, N. Loktionova ${ }^{26}$, R. Lopez-Fernandez ${ }^{24}$, V. Lubimov ${ }^{25}$, L. Lytkin ${ }^{10}$, A. Makankine ${ }^{10}$, E. Malinovski ${ }^{26}$, P. Marage ${ }^{4,5}$, L. Marti ${ }^{12}$, H.-U. Martyn ${ }^{1}$, S.J. Maxfield ${ }^{19}$, A. Mehta ${ }^{19}$, A.B. Meyer $^{12}$, H. Meyer $^{12}$, H. Meyer ${ }^{38}$, J. Meyer $^{12}$, S. Mikocki ${ }^{8}$, I. Milcewicz-Mika ${ }^{8}$, F. Moreau ${ }^{29}$, A. Morozov ${ }^{10}$, J.V. Morris ${ }^{7}$, M.U. Mozer ${ }^{4,5}$, M. Mudrinic ${ }^{2}$, K. Müller ${ }^{42}$, P. Murín ${ }^{17, d}$, Th. Naumann ${ }^{40}$, P.R. Newman ${ }^{3}$, C. Niebuhr ${ }^{12}$, A. Nikiforov ${ }^{12}$, D. Nikitin ${ }^{10}$, G. Nowak ${ }^{8}$, K. Nowak ${ }^{42}$, J.E. Olsson ${ }^{12}$, S. Osman Os. Ozerov $^{25}$, P. Pahl ${ }^{12}$, V. Palichik ${ }^{10}$, I. Panagoulias ${ }^{12, b, w}$, M. Pandurovic ${ }^{2}$, Th. Papadopoulou ${ }^{12, b, w}$, C. Pascaud ${ }^{28}$, G.D. Patel $^{19}$,

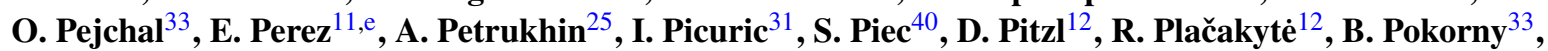
R. Polifka ${ }^{33}$, B. Povh ${ }^{14}$, V. Radescu ${ }^{15}$, A.J. Rahmat ${ }^{19}$, N. Raicevic ${ }^{31}$, A. Raspiareza ${ }^{27}$, T. Ravdandorj $^{36}$, P. Reimer ${ }^{32}$,

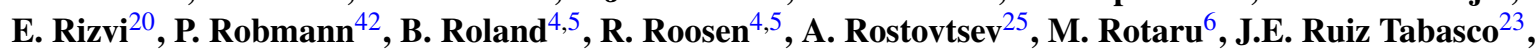
S. Rusakov ${ }^{26}$, D. Š́lek ${ }^{33}$, D.P.C. Sankey ${ }^{7}$, M. Sauter ${ }^{15}$, E. Sauvan ${ }^{22}$, S. Schmitt ${ }^{12}$, L. Schoeffel ${ }^{11}$, A. Schöning ${ }^{15}$, H.-C. Schultz-Coulon ${ }^{16}$, F. Sefkow ${ }^{12}$, R.N. Shaw-West ${ }^{3}$, L.N. Shtarkov ${ }^{26}$, S. Shushkevich ${ }^{27}$, T. Sloan $^{18}$, I. Smiljanic ${ }^{2}$, Y. Soloviev ${ }^{26}$, P. Sopicki ${ }^{8}$, D. South ${ }^{9}$, V. Spaskov ${ }^{10}$, A. Specka ${ }^{29}$, Z. Staykova ${ }^{12}$, M. Steder ${ }^{12}$, B. Stella ${ }^{34}$, G. Stoicea ${ }^{6}$, U. Straumann ${ }^{42}$, D. Sunar ${ }^{12}$, T. Sykora ${ }^{4,5}$, V. Tchoulakov ${ }^{10}$, G. Thompson $^{20}$, P.D. Thompson ${ }^{3}$, T. Toll ${ }^{13}$, F. Tomasz ${ }^{17}$,

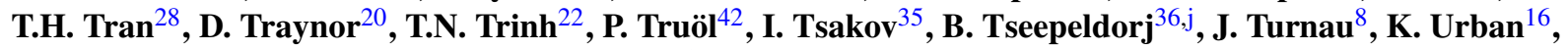
A. Valkárová ${ }^{33}$, C. Vallée ${ }^{22}$, P. Van Mechelen ${ }^{4,5}$, A. Vargas Trevino ${ }^{12}$, Y. Vazdik ${ }^{26}$, S. Vinokurova ${ }^{12}$, V. Volchinski ${ }^{39}$, M. von den Driesch ${ }^{12}$, D. Wegener ${ }^{9}$, C. Wissing ${ }^{12}$, E. Wünsch ${ }^{12}$, J. Žáček ${ }^{33}$, J. Zálešák ${ }^{32}$, Z. Zhang $^{28}$, A. Zhokin ${ }^{25}$, T. Zimmermann ${ }^{41}$, H. Zohrabyan ${ }^{39}$, F. Zomer Zo $^{28}$

\footnotetext{
${ }^{1}$ I. Physikalisches Institut der RWTH, Aachen, Germany

${ }^{2}$ Vinca Institute of Nuclear Sciences, Belgrade, Serbia

${ }^{3}$ School of Physics and Astronomy, University of Birmingham, Birmingham, $\mathrm{UK}^{\mathrm{m}}$

${ }^{4}$ Inter-University Institute for High Energies ULB-VUB, Brussels, Belgium ${ }^{\text {n }}$

${ }^{5}$ Universiteit Antwerpen, Antwerpen, Belgium ${ }^{\mathrm{n}}$

${ }^{6}$ National Institute for Physics and Nuclear Engineering (NIPNE), Bucharest, Romania

${ }^{7}$ Rutherford Appleton Laboratory, Chilton, Didcot, $\mathrm{UK}^{\mathrm{m}}$

${ }^{8}$ Institute for Nuclear Physics, Cracow, Poland ${ }^{\circ}$

${ }^{9}$ Institut für Physik, TU Dortmund, Dortmund, Germany

${ }^{10}$ Joint Institute for Nuclear Research, Dubna, Russia

${ }^{11}$ CEA DSM/Irfu, CE-Saclay, Gif-sur-Yvette, France

${ }^{12}$ DESY, Hamburg, Germany

${ }^{13}$ Institut für Experimentalphysik, Universität Hamburg, Hamburg, Germany ${ }^{1}$
} 
${ }^{14}$ Max-Planck-Institut für Kernphysik, Heidelberg, Germany

${ }^{15}$ Physikalisches Institut, Universität Heidelberg, Heidelberg, Germany ${ }^{1}$

${ }^{16}$ Kirchhoff-Institut für Physik, Universität Heidelberg, Heidelberg, Germany ${ }^{1}$

${ }^{17}$ Institute of Experimental Physics, Slovak Academy of Sciences, Košice, Slovak Republic ${ }^{q}$

${ }^{18}$ Department of Physics, University of Lancaster, Lancaster, UK ${ }^{\mathrm{m}}$

${ }^{19}$ Department of Physics, University of Liverpool, Liverpool, $\mathrm{UK}^{\mathrm{m}}$

${ }^{20}$ Queen Mary and Westfield College, London, UK ${ }^{\mathrm{m}}$

${ }^{21}$ Physics Department, University of Lund, Lund, Sweden ${ }^{\mathrm{r}}$

${ }^{22}$ CPPM, CNRS/IN2P3, Univ. Mediterranee, Marseille, France

${ }^{23}$ Departamento de Fisica Aplicada, CINVESTAV, Mérida, Yucatán, Mexico ${ }^{\mathrm{u}}$

${ }^{24}$ Departamento de Fisica, CINVESTAV IPN, Mexico City, Mexico ${ }^{\mathrm{u}}$

${ }^{25}$ Institute for Theoretical and Experimental Physics, Moscow, Russiav

${ }^{26}$ Lebedev Physical Institute, Moscow, Russia ${ }^{p}$

${ }^{27}$ Max-Planck-Institut für Physik, Munich, Germany

${ }^{28}$ LAL, University Paris-Sud, CNRS/IN2P3, Orsay, France

${ }^{29}$ LLR, Ecole Polytechnique, CNRS/IN2P3, Palaiseau, France

${ }^{30}$ LPNHE, Universités Paris VI and VII, CNRS/IN2P3, Paris, France

${ }^{31}$ Faculty of Science, University of Montenegro, Podgorica, Montenegro ${ }^{\mathrm{p}}$

${ }^{32}$ Institute of Physics, Academy of Sciences of the Czech Republic, Praha, Czech Republic ${ }^{\mathrm{s}}$

${ }^{33}$ Faculty of Mathematics and Physics, Charles University, Praha, Czech Republic ${ }^{\mathrm{s}}$

${ }^{34}$ Dipartimento di Fisica, Università di Roma Tre and INFN Roma 3, Rome, Italy

${ }^{35}$ Institute for Nuclear Research and Nuclear Energy, Sofia, Bulgaria ${ }^{p}$

${ }^{36}$ Institute of Physics and Technology, Mongolian Academy of Sciences, Ulaanbaatar, Mongolia

${ }^{37}$ Paul Scherrer Institut, Villigen, Switzerland

${ }^{38}$ Fachbereich C, Universität Wuppertal, Wuppertal, Germany

${ }^{39}$ Yerevan Physics Institute, Yerevan, Armenia

${ }^{40}$ DESY, Zeuthen, Germany

${ }^{41}$ Institut für Teilchenphysik, ETH, Zürich, Switzerland ${ }^{t}$

${ }^{42}$ Physik-Institut, Universität Zürich, Zürich, Switzerland ${ }^{t}$

Received: 4 January 2010 / Revised: 14 May 2010 / Published online: 16 July 2010

(C) The Author(s) 2010. This article is published with open access at Springerlink.com

a e-mail: kruegerk@mail.desy.de

${ }^{\mathrm{b}}$ Also at Physics Department, National Technical University, Zografou Campus, GR-15773 Athens, Greece.

${ }^{\mathrm{c}}$ Also at Rechenzentrum, Universität Wuppertal, Wuppertal, Germany.

${ }^{\mathrm{d}}$ Also at University of P.J. Šafárik, Košice, Slovak Republic.

eAlso at CERN, Geneva, Switzerland.

${ }^{\mathrm{f}}$ Also at Max-Planck-Institut für Physik, München, Germany.

${ }^{\mathrm{g}}$ Also at Comenius University, Bratislava, Slovak Republic.

${ }^{\mathrm{h}}$ Also at DESY and University Hamburg, Helmholtz Humboldt Research Award.

${ }^{\mathrm{i}}$ Also at Faculty of Physics, University of Bucharest, Bucharest, Romania.

${ }^{\mathrm{j}}$ Also at Ulaanbaatar University, Ulaanbaatar, Mongolia.

${ }^{\mathrm{k}}$ Supported by the Initiative and Networking Fund of the Helmholtz Association (HGF) under the contract VH-NG-401.

${ }^{\text {l}}$ Supported by the Bundesministerium für Bildung und Forschung, FRG, under contract numbers 05H09GUF, 05H09VHC, 05H09VHF, 05H16PEA.

${ }^{\mathrm{m}}$ Supported by the UK Science and Technology Facilities Council, and formerly by the UK Particle Physics and Astronomy Research Council.

${ }^{\mathrm{n}}$ Supported by FNRS-FWO-Vlaanderen, IISN-IIKW and IWT and by Interuniversity Attraction Poles Programme, Belgian Science Policy.

${ }^{\circ}$ Partially Supported by Polish Ministry of Science and Higher Education, grant PBS/DESY/70/2006.

${ }^{\mathrm{p}}$ Supported by the Deutsche Forschungsgemeinschaft.
Abstract The production of leading neutrons, where the neutron carries a large fraction $x_{L}$ of the incoming proton's longitudinal momentum, is studied in deep-inelastic positron-proton scattering at HERA. The data were taken with the H1 detector in the years 2006 and 2007 and correspond to an integrated luminosity of $122 \mathrm{pb}^{-1}$. The semiinclusive cross section is measured in the phase space defined by the photon virtuality $6<Q^{2}<100 \mathrm{GeV}^{2}$, Bjorken scaling variable $1.5 \cdot 10^{-4}<x<3 \cdot 10^{-2}$, longitudinal momentum fraction $0.32<x_{L}<0.95$ and neutron transverse momentum $p_{T}<0.2 \mathrm{GeV}$. The leading neutron structure function, $F_{2}^{L N(3)}\left(Q^{2}, x, x_{L}\right)$, and the fraction of deepinelastic scattering events containing a leading neutron are studied as a function of $Q^{2}, x$ and $x_{L}$. Assuming that the

${ }^{\mathrm{q}}$ Supported by VEGA SR grant no. 2/7062/ 27.

${ }^{\mathrm{r}}$ Supported by the Swedish Natural Science Research Council.

${ }^{\mathrm{s}}$ Supported by the Ministry of Education of the Czech Republic under the projects LC527, INGO-1P05LA259 and MSM0021620859.

${ }^{\text {t}}$ Supported by the Swiss National Science Foundation.

"Supported by CONACYT, México, grant 48778-F.

${ }^{\mathrm{v}}$ Russian Foundation for Basic Research (RFBR), grant no 1329.2008.2.

${ }^{\mathrm{w}}$ This project is co-funded by the European Social Fund $(75 \%)$ and National Resources (25\%) - (EPEAEK II) - PYTHAGORAS II. 
pion exchange mechanism dominates leading neutron production, the data provide constraints on the shape of the pion structure function.

\section{Introduction}

The production of leading baryons in deep-inelastic scattering (DIS) provides a testing ground for the theory of strong interactions in the soft regime. Events containing a neutron, which carries a large fraction $x_{L}$ of the longitudinal momentum of the incoming proton, have been observed in electronproton collisions at HERA [1-7]. The generic process giving rise to leading neutron production, $e p \rightarrow e^{\prime} n X$, is illustrated in Fig. 1a. These energetic neutrons can be produced in the fragmentation of the proton remnant. However, the pion exchange mechanism, illustrated in Fig. 1b, is expected to dominate leading neutron production at large $x_{L}$ and low transverse momentum of the neutron [8-14]. In this picture of leading neutron production, the proton fluctuates into a state consisting of a positively charged pion and a neutron $p \rightarrow n \pi^{+}$. The virtual photon subsequently interacts with a parton from the pion. Consequently, the cross section factorises into two parts (proton vertex factorisation): one factor describes the proton fluctuation into a $n \pi^{+}$state, the other describes the photon-pion scattering $[8,9]$. Therefore, assuming proton vertex factorisation, the production of leading neutrons in DIS at HERA provides supplementary constraints on the structure of the pion at low to medium Bjorken- $x$, as the knowledge of the pion structure from fixed target experiments [15-20] is limited to higher $x$ values. Previous $\mathrm{H} 1$ and ZEUS studies of semi-inclusive leading neutron DIS cross sections $[1,2]$ demonstrate that these measurements are indeed sensitive to the structure of the pion and can distinguish between different parameterisations of the pion structure function.

The comparison of leading neutron production with inclusive DIS provides tests of fragmentation mechanisms. The hypothesis of limiting fragmentation [21,22] states that, in the high-energy limit, the cross section for the inclusive production of particles in the target fragmentation region becomes independent of the incident projectile energy.
This hypothesis implies that, in DIS, leading neutron production is insensitive to Bjorken- $x$ and the virtuality of the exchanged photon $Q^{2}$.

In this paper a measurement of the semi-inclusive cross section for leading neutron production in DIS is presented. This analysis is based on a data sample corresponding to an integrated luminosity which is 36 times larger than that of the previous $\mathrm{H} 1$ publication [1]. A new neutron calorimeter with improved performance is used. The larger data set together with better experimental capabilities allow the extension of the kinematic range of the measurement to higher values of $Q^{2}$ and $x$ and an increased experimental precision of the measurements.

\section{Event kinematics and reconstruction}

The kinematics of semi-inclusive leading neutron production are shown in Fig. 1a, where the four-vectors of the incoming and outgoing particles and of the exchanged virtual photon $\gamma^{*}$ are indicated. The kinematic variables $Q^{2}, x$ and $y$ are used to describe the inclusive DIS scattering process. They are defined as

$Q^{2}=-q^{2}, \quad x=\frac{Q^{2}}{2 p \cdot q}, \quad y=\frac{p \cdot q}{p \cdot k}$,

where $p, k$ and $q$ are the four-momenta of the incident proton, the incident positron and the exchanged virtual photon, respectively. These variables are reconstructed using the technique introduced in [23], which optimises the resolution throughout the measured $y$ range by exploiting information from both the scattered positron and the hadronic final state.

The kinematic variables used to describe the final state neutron are the longitudinal momentum fraction $x_{L}$ and the squared four-momentum transfer $t$ between the incident proton and the final state neutron

$$
\begin{aligned}
& x_{L}=1-\frac{q \cdot\left(p-p_{n}\right)}{q \cdot p} \simeq E_{n} / E_{p}, \\
& t=\left(p-p_{n}\right)^{2} \simeq-\frac{p_{T}^{2}}{x_{L}}-\left(1-x_{L}\right)\left(\frac{m_{n}^{2}}{x_{L}}-m_{p}^{2}\right),
\end{aligned}
$$

Fig. 1 (a) Generic diagram for leading neutron production $e p \rightarrow e^{\prime} n X$ in deep-inelastic scattering. (b) Diagram of the same process assuming that it proceeds via pion exchange
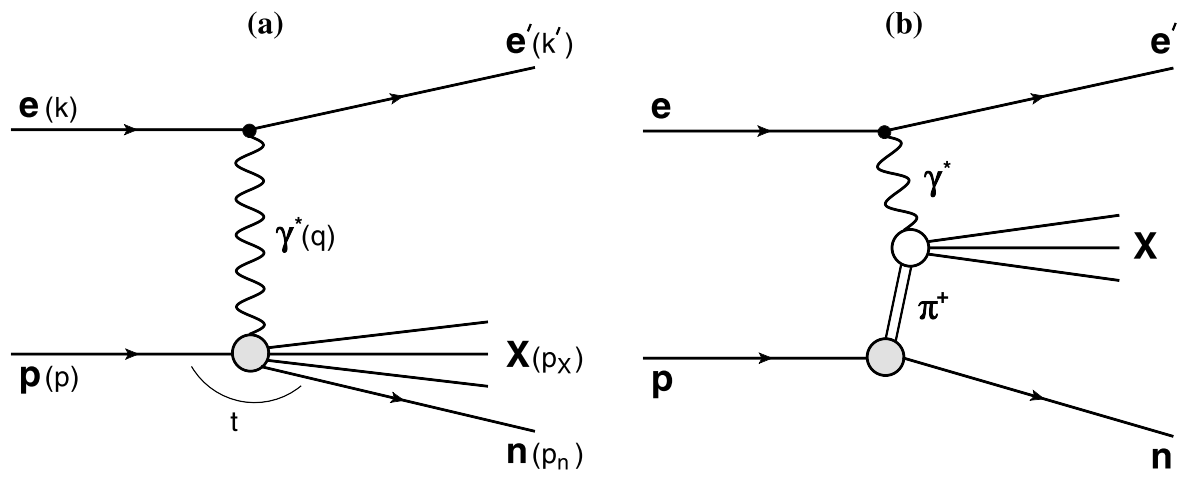
where $m_{p}$ is the proton mass, $p_{n}$ is the four-momentum of the final state neutron, $m_{n}$ is the neutron mass and $E_{n}$ and $p_{T}$ are the neutron energy and transverse momentum, respectively.

The four-fold differential cross section for leading neutron production can be parameterised by a semi-inclusive structure function, $F_{2}^{L N(4)}$, defined by

$$
\begin{aligned}
& \frac{\mathrm{d}^{4} \sigma(e p \rightarrow e n X)}{\mathrm{d} x \mathrm{~d} Q^{2} \mathrm{~d} x_{L} \mathrm{~d} t} \\
& \quad=\frac{4 \pi \alpha^{2}}{x Q^{4}}\left(1-y+\frac{y^{2}}{2}\right) F_{2}^{L N(4)}\left(Q^{2}, x, x_{L}, t\right) .
\end{aligned}
$$

The contribution from longitudinally polarised photons can be neglected in the phase space studied in this analysis. Integrating (3) over $t$ yields the semi-inclusive structure function $F_{2}^{L N(3)}$ measured in this analysis

$$
\begin{aligned}
& \frac{\mathrm{d}^{3} \sigma(e p \rightarrow e n X)}{\mathrm{d} x \mathrm{~d} Q^{2} \mathrm{~d} x_{L}} \\
& =\int_{t_{0}}^{t_{\min }} \frac{\mathrm{d}^{4} \sigma(e p \rightarrow e n X)}{\mathrm{d} x \mathrm{~d} Q^{2} \mathrm{~d} x_{L} \mathrm{~d} t} \mathrm{~d} t \\
& =\frac{4 \pi \alpha^{2}}{x Q^{4}}\left(1-y+\frac{y^{2}}{2}\right) F_{2}^{L N(3)}\left(Q^{2}, x, x_{L}\right),
\end{aligned}
$$

where the integration limits are

$$
\begin{aligned}
& t_{\min }=-\left(1-x_{L}\right)\left(\frac{m_{n}^{2}}{x_{L}}-m_{p}^{2}\right) \text { and } \\
& t_{0}=-\frac{\left(p_{T}^{\max }\right)^{2}}{x_{L}}+t_{\min } .
\end{aligned}
$$

Here, $p_{T}^{\max }$ is the upper limit of the neutron transverse momentum used for the $F_{2}^{L N(3)}$ measurement. Smaller values of $p_{T}^{\max }$ are expected to enhance the relative contribution of pion exchange $[10,11]$. In this analysis, $p_{T}^{\max }$ is set to $0.2 \mathrm{GeV}$ as in the previous $\mathrm{H} 1$ publication [1].

\section{Experimental procedure and data analysis}

The data used in this analysis were collected with the H1 detector at HERA in the years 2006 and 2007 and correspond to an integrated luminosity of $122 \mathrm{pb}^{-1}$. During this period HERA collided positrons and protons with energies of $E_{e}=27.6 \mathrm{GeV}$ and $E_{p}=920 \mathrm{GeV}$, respectively.

\section{$3.1 \mathrm{H} 1$ detector}

A detailed description of the $\mathrm{H} 1$ detector can be found elsewhere [24-28]. Here, a brief account is given of the components most relevant to the present analysis. The origin of the right-handed $\mathrm{H} 1$ coordinate system is the nominal ep interaction point. The direction of the proton beam defines the positive $z$-axis (forward direction); the polar angle $\theta$ is measured with respect to this axis. Transverse momenta are measured in the $x-y$ plane.

The $e p$ interaction region is surrounded by a two-layered silicon strip detector and two large concentric drift chambers. Using these detectors charged particle momenta are measured in the angular range $25^{\circ}<\theta<155^{\circ}$ with a resolution of $\sigma\left(p_{T}\right) / p_{T}=0.005 p_{T} / \mathrm{GeV} \oplus 0.015$ [29]. The tracking system is surrounded by a finely segmented Liquid Argon (LAr) calorimeter, which covers a range in polar angle of $4^{\circ}<\theta<154^{\circ}$ with full azimuthal acceptance. The LAr calorimeter consists of an electromagnetic section with lead absorber and a hadronic section with steel absorber. The total depth of the LAr calorimeter ranges from 4.5 to 8 hadronic interaction lengths. Its energy resolution, determined in test beam measurements, is $\sigma(E) / E \approx 12 \% / \sqrt{E[\mathrm{GeV}]} \oplus 1 \%$ for electrons and $\sigma(E) / E \approx 50 \% / \sqrt{E[\mathrm{GeV}]} \oplus 2 \%$ for charged pions $[30,31]$. The backward region $\left(153^{\circ}<\theta<177.8^{\circ}\right)$ is covered by a lead/scintillating-fibre calorimeter, the SpaCal. Its main purpose is the detection of scattered positrons. The energy resolution for positrons is $\sigma(E) / E \approx 7.1 \% /$ $\sqrt{E[\mathrm{GeV}]} \oplus 1 \%$. The LAr and SpaCal calorimeters are surrounded by a superconducting solenoid which provides a uniform magnetic field of $1.16 \mathrm{~T}$ along the beam direction.

The luminosity is measured via the Bethe-Heitler process $e p \rightarrow e^{\prime} p \gamma$. The final state photon is detected in a dedicated calorimeter situated near the beam pipe at $z=-103 \mathrm{~m}$.

\subsection{Detection of leading neutrons}

Leading neutrons are detected in the forward neutron calorimeter (FNC). The FNC is situated at a polar angle of $0^{\circ}$ beyond the magnets used to deflect the proton beam, at $z=106 \mathrm{~m}$. A schematic view of the FNC is shown in Fig. 2a. It consists of the Main Calorimeter and the Preshower Calorimeter. In addition, two layers of veto counters situated $2 \mathrm{~m}$ in front of the Preshower Calorimeter are used to veto charged particles.

The Preshower Calorimeter is a $40 \mathrm{~cm}$ long lead-scintillator sandwich calorimeter, corresponding to about 60 radiation lengths or 1.6 hadronic interaction lengths. It is composed of 24 planes: the first 12 planes each consist of a lead plate of $7.5 \mathrm{~mm}$ thickness and a scintillator plate of $2.6 \mathrm{~mm}$ thickness, the second 12 planes each consist of a lead plate of $14 \mathrm{~mm}$ thickness and a scintillator plate of $5.2 \mathrm{~mm}$ thickness. The transverse size of the scintillating plates is $26 \times 26 \mathrm{~cm}^{2}$. Each scintillating plate has 45 parallel grooves holding $1.2 \mathrm{~mm}$ diameter wavelength shifter (WLS) fibres. In order to obtain good spatial resolution, the orientation of fibres alternates from horizontal to vertical in consecutive planes. At each plane the fibres are bundled into 
(a)

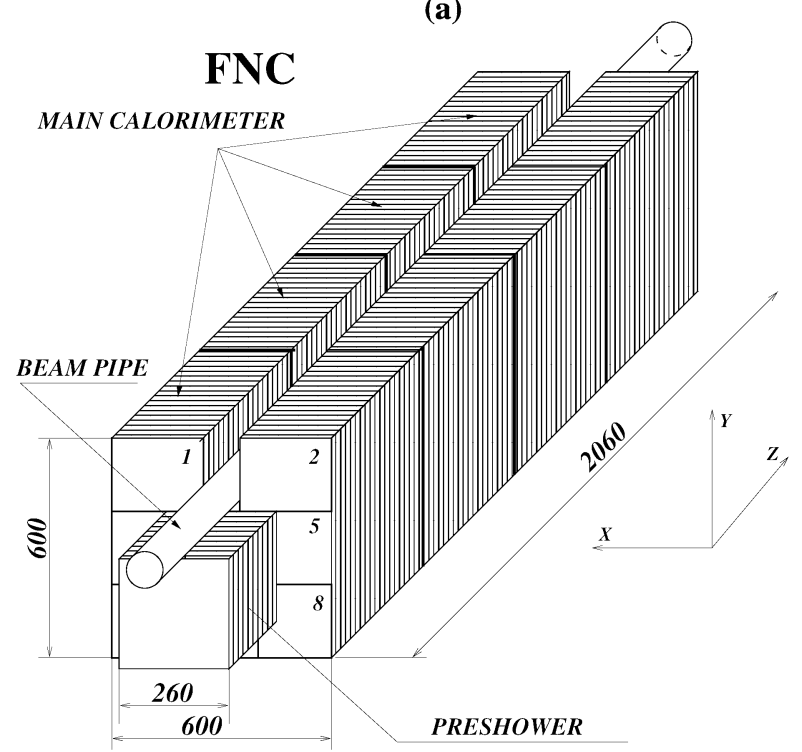

(b)

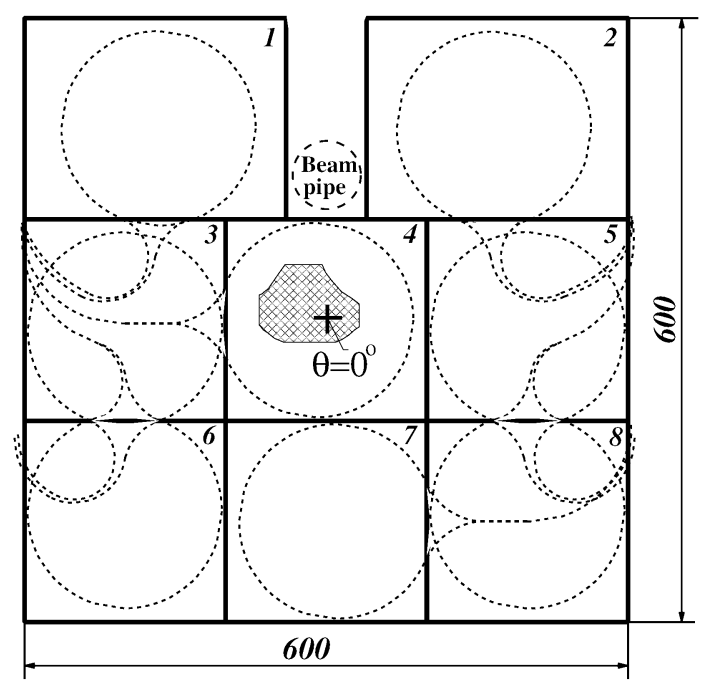

Fig. 2 (a) Schematic view of the FNC. (b) Layout of tiles on an active board of the Main Calorimeter; the position of readout fibres is indicated by dotted lines; the hatched area shows the geometrical

nine strips of five fibres. Longitudinally, the strips are combined into 9 vertical and 9 horizontal towers which are finally connected to 18 photomultipliers.

The Main Calorimeter of the FNC is a sandwich-type calorimeter consisting of four identical sections. Each section is $51.5 \mathrm{~cm}$ long with transverse dimensions of $60 \times 60 \mathrm{~cm}^{2}$ and consists of 25 lead absorber plates of $14 \mathrm{~mm}$ thickness and 25 active boards with $3 \mathrm{~mm}$ thick scintillators. Each active board is made of 6 scintillating tiles with a transverse size of $20 \times 20 \mathrm{~cm}^{2}$ and 2 tiles of transverse size $20 \times 26 \mathrm{~cm}^{2}$, as shown in Fig. 2 b. Each scintillating tile has a circular groove holding a $1 \mathrm{~mm}$ diameter WLS fibre which is attached via light connectors to two $1 \mathrm{~mm}$ diameter transparent fibres. Longitudinally, for each section the fibres from 25 tiles with the same transverse position are bundled into a tower and connected to one photomultiplier. There are then 8 towers in each section, making a total of 32 towers in the Main Calorimeter. The proton beam pipe is located in a rectangular space along the top of the calorimeter, as can be seen in Fig. 2. The total length of the Main Calorimeter is $206 \mathrm{~cm}$, corresponding to 8.9 hadronic interaction lengths.

All modules of the FNC were initially calibrated at CERN using electron beams with energies between 120 and $230 \mathrm{GeV}$ and hadron beams with energies between 120 and $350 \mathrm{GeV}$. The FNC was positioned on a movable platform which allowed the response of each module and tower to be measured separately. After this initial calibration, the FNC had an approximately uniform response independent of impact position. The linearity of the energy response of the FNC was measured at HERA from acceptance window defined by the beam line elements. The position corresponding to $\theta=0^{\circ}$ is also indicated. All dimensions are given in $\mathrm{mm}$

beam-gas interactions during dedicated runs, when the proton beam was accelerated to five intermediate energies between $150 \mathrm{GeV}$ and $920 \mathrm{GeV}$. The energy response of the FNC is linear to a precision of $3 \%$ and the hadronic energy resolution is $\sigma(E) / E \approx 63 \% / \sqrt{E[\mathrm{GeV}]} \oplus 3 \%$. The energy resolution for electromagnetic showers, which are always fully contained in the Preshower Calorimeter, is $\sigma(E) / E \approx 20 \% / \sqrt{E[\mathrm{GeV}]}$. For hadronic showers starting in the Main Calorimeter, the spatial resolution is $\sigma(x, y) \approx$ $10 \mathrm{~cm} / \sqrt{E[\mathrm{GeV}]} \oplus 0.6 \mathrm{~cm}$. A better spatial resolution of about $2 \mathrm{~mm}$ is achieved for the electromagnetic showers and for those hadronic showers starting in the Preshower Calorimeter.

The acceptance of the FNC is defined by the aperture of the HERA beam line magnets and is limited to neutron scattering angles of $\theta_{n} \lesssim 0.8 \mathrm{mrad}$ with an approximately $30 \%$ azimuthal coverage. The geometrical acceptance window of the FNC is indicated in Fig. $2 b$.

After the calorimeter was installed in the $\mathrm{H} 1$ beam line, the stability of the calibration constants was monitored using interactions between the proton beam and residual gas molecules in the beam pipe. The neutron energy spectrum was compared with the results of a Monte Carlo simulation based on pion exchange. From this monitoring, the time dependent variations of the calibration constants were determined to be of order a few per cent. Short term variations of photomultiplier gain were monitored using LED signals. The LEDs were operated during empty bunches at a frequency of $0.8 \mathrm{~Hz}$. The averaged LED signal responses were used to provide offline energy corrections applied during the reconstruction. 
The longitudinal segmentation of the FNC allows efficient discrimination of neutrons from charged particles or electromagnetic energy deposits. Charged particles are rejected using signals from the veto counters. Events with an energy deposit only in the Preshower Calorimeter are identified as electromagnetic showers (photons, $\pi^{0}$ mesons). All other patterns of energy deposition are identified as hadronic showers, which are subsequently classified as either those starting in the Preshower Calorimeter or those starting in the Main Calorimeter, the latter having worse spatial resolution. In this analysis both hadronic shower types are used.

\subsection{Event selection}

The data sample was collected using a trigger which requires the scattered positron to be measured in the $\mathrm{SpaCal}$ and at least $100 \mathrm{GeV}$ energy to be deposited in the FNC. The trigger signal from the FNC is formed using an analogue sum of signals from the Preshower Calorimeter and the central towers of the Main Calorimeter. The trigger efficiency is above $97 \%$ in most of the analysis phase space, decreasing to $94 \%$ for $x_{L}<0.4$.

The selection of DIS events is based on the identification of the scattered positron as the most energetic compact calorimetric deposit in the SpaCal with an energy $E_{e}^{\prime}>11 \mathrm{GeV}$ and a polar angle $156^{\circ}<\theta_{e}^{\prime}<175^{\circ}$. The energy weighted cluster radius is required to be less than $4 \mathrm{~cm}$, as expected for an electromagnetic shower [32]. The $z$-coordinate of the primary event vertex is required to be within $\pm 35 \mathrm{~cm}$ of the nominal position of the interaction point. The remaining clusters in the calorimeters and the charged tracks are combined to reconstruct the hadronic final state. To suppress events with initial state hard photon radiation, as well as events originating from non-ep interactions, the quantity $E-p_{z}$, summed over all reconstructed particles including the positron, is required to lie between $35 \mathrm{GeV}$ and $70 \mathrm{GeV}$. This quantity is expected to be twice the electron beam energy for DIS events without QED radiation. Furthermore, events are selected within the kinematic range $6<Q^{2}<100 \mathrm{GeV}^{2}, 0.02<y<0.6$ and $1.5 \cdot 10^{-4}<x<3 \cdot 10^{-2}$.

Events containing a leading neutron are selected by requiring a hadronic cluster in the FNC with an energy above $275 \mathrm{GeV}$ and a polar angle below $0.75 \mathrm{mrad}$. The cut on polar angle, defined by the geometrical acceptance of the FNC, restricts the neutron transverse momenta $p_{T}$ to the range $p_{T}<x_{L} \cdot 0.69 \mathrm{GeV}$.

The final data sample contains 315960 events which satisfy these selection criteria. For the measurement of $F_{2}^{L N(3)}$, an additional requirement on the transverse momentum of the neutron $p_{T}<0.2 \mathrm{GeV}$ is applied to enhance the relative contribution of pion exchange and to avoid having an $x_{L}$ dependent $p_{T}$ cut. The number of events selected with this additional requirement is 209150.

\subsection{Monte Carlo simulation and corrections to the data}

Monte Carlo simulations are used to correct the data for the effects of detector acceptance, inefficiencies and migrations between measurement intervals due to finite resolution and QED radiation. All generated events are passed through a GEANT3 [33] based simulation of the H1 apparatus and are processed using the same reconstruction and analysis framework as is used for the data.

The DJANGO [34] program generates inclusive DIS events. It is based on leading order electroweak cross sections and takes QCD effects into account up to order $\alpha_{s}$. The hadronic final state is simulated using ARIADNE [35], based on the Colour Dipole Model, with subsequent hadronisation effects modelled using the Lund string fragmentation model as implemented in JETSET [36]. DJANGO is also used in this analysis to simulate events where leading neutrons originate from proton remnant fragmentation. RAPGAP [37] is a general purpose event generator for inclusive and diffractive $e p$ interactions. Higher order QCD effects are simulated using parton showers and the final state hadrons are obtained via Lund string fragmentation. Higher order electroweak processes in the DJANGO and RAPGAP generators are simulated using an interface to HERACLES [38].

In the version denoted below as RAPGAP- $\pi$, the program simulates exclusively the scattering of virtual or real photons off an exchanged pion. Here, the cross section for photon-proton scattering to the final state $n X$ takes the form

$\mathrm{d} \sigma\left(e p \rightarrow e^{\prime} n X\right)=f_{\pi^{+} / p}\left(x_{L}, t\right) \cdot \mathrm{d} \sigma\left(e \pi^{+} \rightarrow e^{\prime} X\right)$,

where $f_{\pi^{+} / p}\left(x_{L}, t\right)$ represents the pion flux associated with the splitting of a proton into a $\pi^{+} n$ system and $\mathrm{d} \sigma\left(e \pi^{+} \rightarrow e^{\prime} X\right)$ is the cross section of the positron-pion interaction. There are several parameterisations of the pion flux [9-13]. In this analysis, the pion flux factor is taken from the light-cone representation [10] as

$$
\begin{aligned}
f_{\pi^{+} / p}\left(x_{L}, t\right)= & \frac{1}{2 \pi} \frac{g_{p \pi n}^{2}}{4 \pi}\left(1-x_{L}\right) \frac{-t}{\left(m_{\pi}^{2}-t\right)^{2}} \\
& \times \exp \left(-R_{\pi n}^{2} \frac{m_{\pi}^{2}-t}{1-x_{L}}\right),
\end{aligned}
$$

where $m_{\pi}$ is the pion mass, $g_{p \pi n}^{2} / 4 \pi=13.6$ is the $p \pi n$ coupling constant deduced from phenomenological analyses of low-energy data [39] and $R_{\pi n}=0.93 \mathrm{GeV}^{-1}$ is the radius of the pion-proton Fock state [10].

The DJANGO and RAPGAP- $\pi$ Monte Carlo simulations are calculated using GRV leading order parton distributions for the proton [40] and the pion [41], respectively.

Figure 3 shows the observed energy and $p_{T}$ distribution of the neutron for the selected data sample together 
Fig. 3 The observed neutron energy (a) and transverse momentum (b) distributions in the kinematic range $6<Q^{2}<100 \mathrm{GeV}^{2}$ and $1.5 \cdot 10^{-4}<x<3 \cdot 10^{-2}$. The data are compared to the predictions of RAPGAP- $\pi$ (dashed line) and DJANGO (dotted line) Monte Carlo simulations. Also shown is a weighted combination of those two simulations (full line), as described in Sect. 3.4
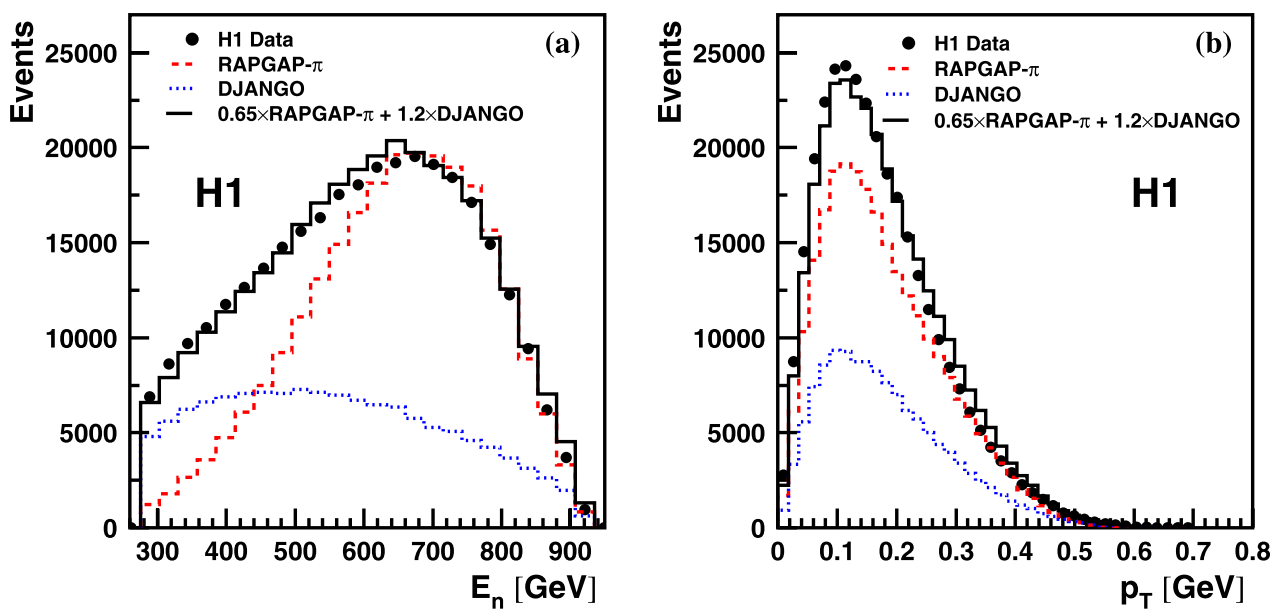

Table 1 Bins in $Q^{2}, x$ and $x_{L}$ as used for the measurement of the semi-inclusive structure function $F_{2}^{L N(3)}$

\begin{tabular}{ll|ll|ll}
\hline$Q^{2}$-bin & $Q^{2}$ range $\left[\mathrm{GeV}^{2}\right]$ & $x$-bin & $x$ range & $x_{L}$-bin & $x_{L}$ range \\
\hline 1 & $6.00 \div 9.00$ & 1 & $1.50 \cdot 10^{-4} \div 3.20 \cdot 10^{-4}$ & 1 & $0.32 \div 0.41$ \\
2 & $9.00 \div 13.5$ & 2 & $3.20 \cdot 10^{-4} \div 6.82 \cdot 10^{-4}$ & 2 & $0.41 \div 0.50$ \\
3 & $13.5 \div 20.0$ & 3 & $6.82 \cdot 10^{-4} \div 1.45 \cdot 10^{-3}$ & 3 & $0.50 \div 0.59$ \\
4 & $20.0 \div 30.0$ & 4 & $1.45 \cdot 10^{-3} \div 3.10 \cdot 10^{-3}$ & 4 & $0.59 \div 0.68$ \\
5 & $30.0 \div 45.0$ & 5 & $3.10 \cdot 10^{-3} \div 6.60 \cdot 10^{-3}$ & 5 & $0.68 \div 0.77$ \\
6 & $45.0 \div 67.0$ & 6 & $6.60 \cdot 10^{-3} \div 1.41 \cdot 10^{-2}$ & 6 & $0.77 \div 0.86$ \\
7 & $67.0 \div 100$ & 7 & $1.41 \cdot 10^{-2} \div 3.00 \cdot 10^{-2}$ & 7 & $0.86 \div 0.95$ \\
\hline
\end{tabular}

with Monte Carlo simulations. The differences between the RAPGAP- $\pi$ and DJANGO generators are particularly visible in the neutron energy distributions (Fig. 3a). The RAPGAP- $\pi$ simulation peaks at $E_{n} \sim 650 \mathrm{GeV}$ and describes the shape of the distribution at high energies. At lower energies $\left(E_{n} \lesssim 600 \mathrm{GeV}\right.$ ) the RAPGAP- $\pi$ simulation does not describe the data. In this region additional physics processes which are expected to contribute significantly are not simulated. In contrast, the DJANGO simulation predicts a large contribution to the cross section in this region. The best description of the data is achieved if the predictions of the RAPGAP- $\pi$ and DJANGO Monte Carlo programs are added, using weighting factors of 0.65 and 1.2 for RAPGAP- $\pi$ and DJANGO, respectively. This Monte Carlo combination is labelled as " $0.65 \times$ RAPGAP- $\pi+1.2 \times$ DJANGO" in the figures and is used to correct the data.

Cross sections at hadron level are determined from the data by applying bin dependent correction factors. These factors are determined from the combination of DJANGO and RAPGAP- $\pi$ Monte Carlo simulations as the ratios of the cross sections obtained from particles at hadron level without QED radiation to the cross section calculated using reconstructed particles and including QED radiation effects. The typical value of these factors is about 1.2 at the highest $x_{L}$ increasing to 4 at the lowest $x_{L}$ due to the non-uniform azimuthal acceptance of the FNC.
The binning in $Q^{2}, x$ and $x_{L}$ used to measure $F_{2}^{L N(3)}$ is given in Table 1 . The bin purities, defined as the fraction of events reconstructed in a particular bin that originated from that bin at hadron level, and the bin stabilities, defined as the fraction of events originating from a particular bin at hadron level that are reconstructed in that bin, are higher than $50 \%$ for $\left(Q^{2}, x\right)$-bins and higher than $60 \%$ for $x_{L}$ bins.

The measured distributions may contain background arising from different sources. The background from photoproduction processes, where the positron is scattered into the backward beam pipe and a particle from the hadronic final state fakes the positron signature in the SpaCal, is estimated using the PHOJET Monte Carlo generator [42]. This background is negligible except at the highest $y$ values where it can reach $6 \%$. Background also arises from the random coincidence of DIS events, causing activity in the central detector, with proton beam-gas interactions, which give a neutron signal in the FNC. This contribution is estimated by combining DIS events with neutrons originating from beam-gas interactions in the bunch-crossings adjacent to the bunchcrossing of the DIS event. It is found to be smaller than $1 \%$. The estimated background contributions are not subtracted from the measurements.

The contribution from proton dissociation, where the leading neutron originates from the decay of a higher mass state, is estimated using an implementation in RAPGAP of 
the dissociation model originally developed for the DIFFVM [43] Monte Carlo generator. This contribution can be up to $30 \%$ at low $x_{L}$ values, but is less than $5 \%$ for $x_{L}>0.7$. It is included in the cross section definition.

\subsection{Systematic uncertainties}

The effects of various systematic uncertainties on the cross section measurements are determined using Monte Carlo simulations, by propagating the corresponding estimated measurement uncertainty through the full analysis chain.

The acceptance of the FNC is defined by the interaction point and the geometry of the HERA magnets and is determined using Monte Carlo simulations. The angular distribution of the neutrons studied in this analysis is sharply peaked in the forward direction. Therefore the acceptance depends critically on small inclinations of the incoming proton beam with respect to its nominal direction. The uncertainty on the position of the neutron impact point is estimated to be $5 \mathrm{~mm}$, which results in a $4.4 \%$ uncertainty in the FNC acceptance. The uncertainty in the neutron detection efficiency and the uncertainty of $2 \%$ on the absolute energy scale of the FNC lead to a systematic error on the cross section of $2 \%$ and $6 \%$, respectively. An additional $0.5 \%$ uncertainty is attributed to the trigger efficiency. These effects are strongly correlated between measurement intervals and mainly contribute to the overall normalisation uncertainty.

The uncertainties on the measurements of the scattered positron energy (1\%) and angle (1 mrad) in the SpaCal lead to a combined systematic uncertainty of typically $1.9 \%$ on the cross section. The uncertainty of the energy measurement of the hadronic final state in the central detectors affect the reconstruction of the kinematic variables $y, Q^{2}$ and $x$.

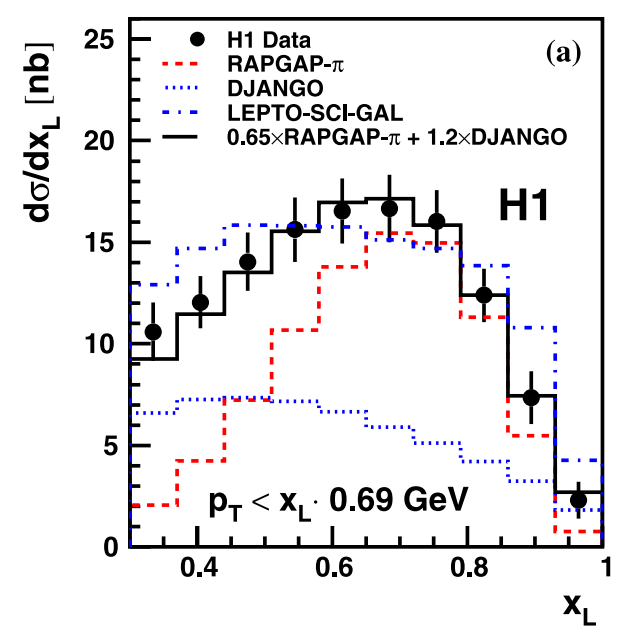

Fig. 4 The cross section as a function of the fractional energy of the neutron $x_{L}$ in the kinematic range $6<Q^{2}<100 \mathrm{GeV}^{2}$ and 1.5 . $10^{-4}<x<3 \cdot 10^{-2}$. The transverse momentum of the neutron is restricted to $p_{T}<x_{L} \cdot 0.69 \mathrm{GeV}$ (a) and $p_{T}<0.2 \mathrm{GeV}(\mathbf{b})$. The data are
This hadronic energy scale uncertainty is estimated to be $4 \%$ for this measurement, leading to an uncertainty on the cross section of $0.4 \%$ on average.

The systematic error on the efficiency to reconstruct the event vertex is determined by comparing the reconstruction efficiencies for the data and the Monte Carlo simulation. The discrepancy is less than $1 \%$.

The systematic uncertainty arising from the radiative corrections and the model dependence of the data correction are estimated by varying the DJANGO and RAPGAP- $\pi$ scaling factors described in Sect. 3.4 within values permitted by the data. The resulting uncertainty on the cross section is below $5 \%$ in most of the bins and typically $2 \%$.

The luminosity measurement uncertainty for the selected run period leads to an overall normalisation uncertainty of $5 \%$.

The total systematic error in each bin is calculated as the quadratic sum of all contributions. Systematic errors are typically $10 \%$ for $F_{2}^{L N(3)}$ and $14 \%$ for $\mathrm{d} \sigma / \mathrm{d} x_{L}$ measurements.

\section{Results}

The single differential leading neutron DIS cross sections as a function of $x_{L}$ are presented in Fig. 4 and Table 2. In Fig. $4 \mathrm{~b}$ the differential cross section in $x_{L}$ is shown for $p_{T}<0.2 \mathrm{GeV}$. The difference between the shapes of distributions in Fig. 4a and Fig. 4b, in particular a steep fall at low $x_{L}$ in Fig. 4a, is due to the geometrical acceptance of the FNC which restricts the accessible $p_{T}$ range to $p_{T}<x_{L} \cdot 0.69 \mathrm{GeV}$.

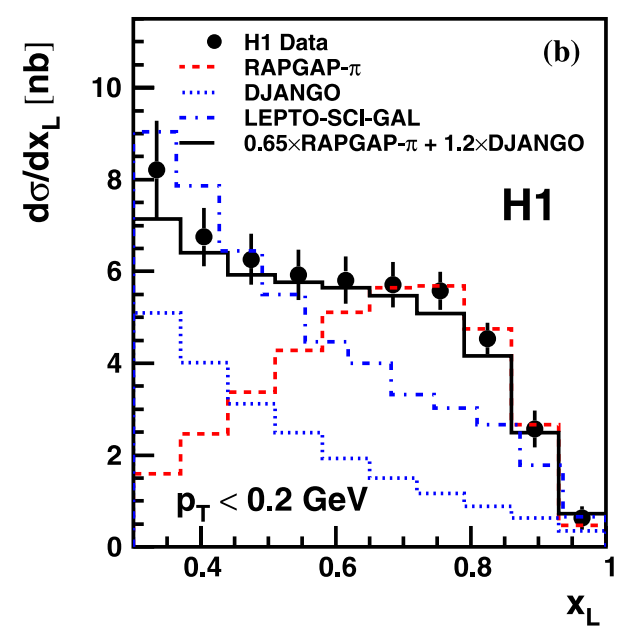

compared to the predictions of RAPGAP- $\pi$ (dashed line), DJANGO (dotted line) and LEPTO-SCI-GAL (dash-dotted line) Monte Carlo simulations. Also shown is a weighted combination of RAPGAP- $\pi$ and DJANGO simulations (full line), as described in Sect. 3.4 
Table 2 Differential cross section $\mathrm{d} \sigma / \mathrm{d} x_{L}$ of leading neutron production in deep-inelastic scattering in the kinematic range

$6<Q^{2}<100 \mathrm{GeV}^{2}$,

$1.5 \cdot 10^{-4}<x<3 \cdot 10^{-2}$ and

$0.32<x_{L}<0.95$. The first

uncertainty is statistical and the second systematic.

Normalisation uncertainties of $5 \%$ are not included

\begin{tabular}{lll}
\hline$x_{L}$ range & $\begin{array}{l}\mathrm{d} \sigma / \mathrm{d} x_{L}[\mathrm{nb}] \\
p_{T}<x_{L} \cdot 0.69 \mathrm{GeV}\end{array}$ & $\begin{array}{l}\mathrm{d} \sigma / \mathrm{d} x_{L}[\mathrm{nb}] \\
p_{T}<0.2 \mathrm{GeV}\end{array}$ \\
\hline $0.30 \div 0.37$ & $10.6 \pm 0.11 \pm 1.3$ & $8.21 \pm 0.09 \pm 0.99$ \\
$0.37 \div 0.44$ & $12.0 \pm 0.11 \pm 1.1$ & $6.75 \pm 0.07 \pm 0.53$ \\
$0.44 \div 0.51$ & $14.0 \pm 0.11 \pm 1.3$ & $6.26 \pm 0.06 \pm 0.45$ \\
$0.51 \div 0.58$ & $15.6 \pm 0.11 \pm 1.4$ & $5.92 \pm 0.05 \pm 0.46$ \\
$0.58 \div 0.65$ & $16.5 \pm 0.11 \pm 1.4$ & $5.81 \pm 0.05 \pm 0.42$ \\
$0.65 \div 0.72$ & $16.7 \pm 0.10 \pm 1.4$ & $5.71 \pm 0.05 \pm 0.40$ \\
$0.72 \div 0.79$ & $16.0 \pm 0.10 \pm 1.3$ & $5.57 \pm 0.05 \pm 0.31$ \\
$0.79 \div 0.86$ & $12.4 \pm 0.08 \pm 1.2$ & $4.54 \pm 0.04 \pm 0.25$ \\
$0.86 \div 0.93$ & $7.3 \pm 0.06 \pm 1.2$ & $2.57 \pm 0.03 \pm 0.38$ \\
$0.93 \div 1.00$ & $2.3 \pm 0.03 \pm 0.9$ & $0.63 \pm 0.01 \pm 0.26$ \\
\hline
\end{tabular}

The measured cross sections are compared with the Monte Carlo simulations. For large values of $x_{L} \gtrsim 0.7$, the RAPGAP- $\pi$ simulation describes the shape of the $x_{L}$ distributions well, in agreement with the assumption that at high $x_{L}$ the dominant mechanism for leading neutron production is pion exchange. The full $x_{L}$ range is well described by the sum of the RAPGAP- $\pi$ and DJANGO Monte Carlo generators, using the scaling factors discussed in Sect. 3.4. This indicates that the $\pi$ exchange mechanism dominates at high $x_{L}$, while proton remnant fragmentation gives a significant contribution at low $x_{L}$.

The measured cross sections are also compared with the predictions of the Soft Colour Interaction model (SCI) [44], implemented in the LEPTO Monte Carlo generator program [45]. In the SCI model, the production of leading baryons and diffraction-like configurations is enhanced via non-perturbative colour rearrangements between the outgoing partons. A refined version of the model uses a generalised area law (GAL) [46] for the colour rearrangement probability. Compared to DJANGO MC predictions, SCIGAL improves the description at higher $x_{L}$, as can be seen in Fig. 4. However, for lower $p_{T}$ values the predicted cross section for $x_{L}>0.5$ is still too low.

Figure 5 and Table 3 present the measurement of the semi-inclusive structure function $F_{2}^{L N(3)}\left(Q^{2}, x, x_{L}\right)$ in the range $6<Q^{2}<100 \mathrm{GeV}^{2}, 1.5 \cdot 10^{-4}<x<3 \cdot 10^{-2}$, $0.32<x_{L}<0.95$ and $p_{T}<0.2 \mathrm{GeV}$. In all $\left(Q^{2}, x\right)$ bins, the shape of the $F_{2}^{L N(3)}$ distribution as a function of $x_{L}$ is similar to the shape of the single differential cross section in $x_{L}$ (Fig. 4b). The distributions are reasonably well described by the combination of RAPGAP- $\pi$ and DJANGO simulations.

The measurement of $F_{2}^{L N(3)}$ allows the validity of the hypothesis of limiting fragmentation to be tested, according to which the production of leading neutrons in the proton fragmentation region is independent of $Q^{2}$ and $x$. To investigate this prediction, the ratio of the semi-inclusive structure function $F_{2}^{L N(3)}$ to the proton structure function $F_{2}$ is studied as a function of $Q^{2}$ in bins of $x$ and $x_{L}$ (Fig. 6). The values of
$F_{2}$ are obtained from the H1PDF2009 parameterisation [32]. The horizontal lines indicate the average value of the ratio for a given $x_{L}$ bin. These average values decrease from $7 \%$ to $2 \%$ with increasing $x_{L}$, reflecting the general behaviour observed in Figs. 4 and 5. The ratios are almost independent of $x$ and $Q^{2}$ in each $x_{L}$ bin, implying that $F_{2}^{L N(3)}$ and $F_{2}$ have a similar $\left(Q^{2}, x\right)$ behaviour, as expected from the hypothesis of limiting fragmentation.

Assuming that leading neutrons are produced via the exchange of a colour singlet particle, e.g. the $\pi^{+}$, the structure function $F_{2}^{L N(3)}$ factorises into a flux factor which is a function of $x_{L}$ and a structure function $F_{2}^{L N(2)}$ which depends on $Q^{2}$ and $\beta=x /\left(1-x_{L}\right)$. The quantity $\beta$ can be interpreted as the fraction of the exchanged particle's momentum carried by the parton interacting with the virtual photon. The value of $F_{2}^{L N(3)}\left(Q^{2}, \beta, x_{L}\right)$ is obtained by replacing the variable $x$ with $\beta$ in (4). The distribution of $F_{2}^{L N(3)}$, shown in Fig. 7, has a similar dependence on $\beta$ in all $\left(Q^{2}, x_{L}\right)$ bins. This behaviour can be approximated by a power law function $F_{2}^{L N(3)} \propto \beta^{-\lambda}$. In each $\left(Q^{2}, x_{L}\right)$ bin a fit of the parameter $\lambda$ is performed. Within uncertainties the value of the fitted parameter $\lambda$ is independent of $x_{L}$ which is consistent with proton vertex factorisation.

However, as a function of $Q^{2}$, the values of $\lambda$ increase from 0.23 at lowest $Q^{2}$ to 0.3 at highest $Q^{2}$. This slow $Q^{2}$ dependence is similar to the rise towards low $x$ of the proton structure function $F_{2}$ measured in inclusive DIS [47]. It is further investigated by fitting the measured $F_{2}^{L N(3)}\left(Q^{2}, \beta, x_{L}\right)$ assuming the following functional form

$$
\begin{gathered}
F_{2}^{L N(3)}\left(Q^{2}, \beta, x_{L}\right)=c\left(x_{L}\right) \cdot \beta^{-\lambda\left(Q^{2}\right)} \\
\quad \text { with } \lambda=a \cdot \ln \left(Q^{2} / \Lambda^{2}\right),
\end{gathered}
$$

where $a, \Lambda$ and the normalisations $c\left(x_{L}\right)$ are the free parameters of the fit. This nine parameter fit over the whole $x_{L}$ range has a good $\chi^{2}$, supporting the validity of the employed ansatz (8). Within the experimental uncertainties the 


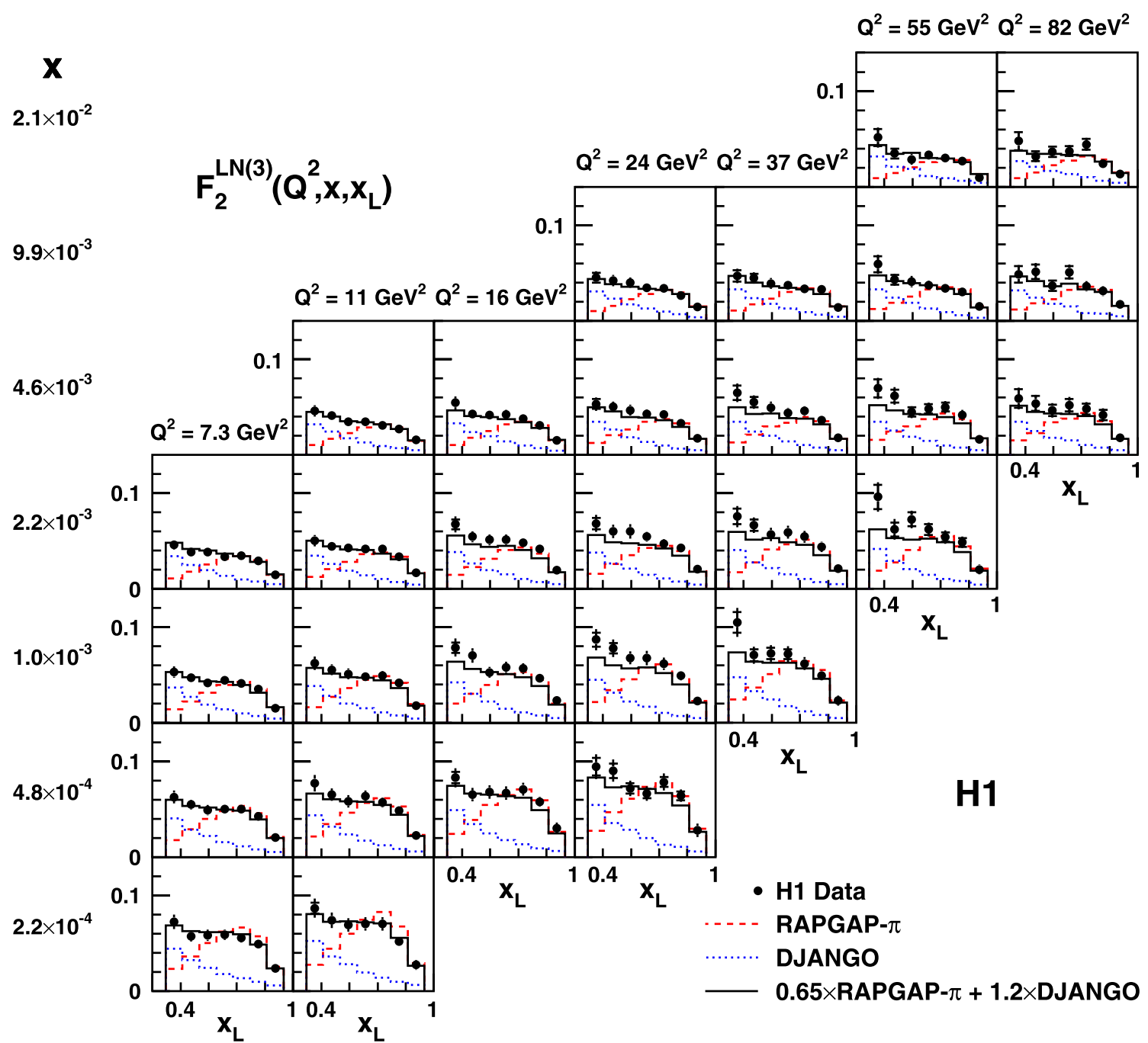

Fig. 5 The semi-inclusive structure function $F_{2}^{L N(3)}\left(Q^{2}, x, x_{L}\right)$, for neutrons with $p_{T}<0.2 \mathrm{GeV}$, compared to the predictions of RAPGAP- $\pi$ (dashed line) and DJANGO (dotted line) Monte Carlo simulations. Also shown is a weighted combination of those two simulations (full line), as described in Sect. 3.4

tical and the second systematic. Normalisation uncertainties of 5\% are not included
Table 3 The semi-inclusive structure function $F_{2}^{L N(3)}\left(Q^{2}, x, x_{L}\right)$, for neutrons with $p_{T}<0.2 \mathrm{GeV}$. The bin centre values of $Q^{2}, x, x_{L}$ and $\beta$ in the corresponding bins are also given. The first uncertainty is statis-

\begin{tabular}{|c|c|c|c|c|}
\hline$Q^{2}\left[\mathrm{GeV}^{2}\right]$ & $x$ & $x_{L}$ & $\beta$ & $F_{2}^{L N(3)}$ \\
\hline 7.3 & $2.24 \cdot 10^{-4}$ & 0.365 & $3.53 \cdot 10^{-4}$ & $0.0724 \pm 0.0035 \pm 0.0058$ \\
\hline 7.3 & $2.24 \cdot 10^{-4}$ & 0.455 & $4.12 \cdot 10^{-4}$ & $0.0573 \pm 0.0024 \pm 0.0044$ \\
\hline 7.3 & $2.24 \cdot 10^{-4}$ & 0.545 & $4.93 \cdot 10^{-4}$ & $0.0582 \pm 0.0022 \pm 0.0049$ \\
\hline 7.3 & $2.24 \cdot 10^{-4}$ & 0.635 & $6.14 \cdot 10^{-4}$ & $0.0589 \pm 0.0020 \pm 0.0042$ \\
\hline 7.3 & $2.24 \cdot 10^{-4}$ & 0.725 & $8.16 \cdot 10^{-4}$ & $0.0557 \pm 0.0018 \pm 0.0036$ \\
\hline 7.3 & $2.24 \cdot 10^{-4}$ & 0.815 & $1.21 \cdot 10^{-3}$ & $0.0489 \pm 0.0017 \pm 0.0023$ \\
\hline 7.3 & $2.24 \cdot 10^{-4}$ & 0.905 & $2.36 \cdot 10^{-3}$ & $0.0236 \pm 0.0012 \pm 0.0043$ \\
\hline 7.3 & $4.78 \cdot 10^{-4}$ & 0.365 & $7.53 \cdot 10^{-4}$ & $0.0627 \pm 0.0030 \pm 0.0052$ \\
\hline 7.3 & $4.78 \cdot 10^{-4}$ & 0.455 & $8.77 \cdot 10^{-4}$ & $0.0547 \pm 0.0022 \pm 0.0037$ \\
\hline
\end{tabular}


Table 3 (Continued)

\begin{tabular}{|c|c|c|c|c|}
\hline$Q^{2}\left[\mathrm{GeV}^{2}\right]$ & $x$ & $x_{L}$ & $\beta$ & $F_{2}^{L N(3)}$ \\
\hline 7.3 & $4.78 \cdot 10^{-4}$ & 0.545 & $1.05 \cdot 10^{-3}$ & $0.0490 \pm 0.0018 \pm 0.0046$ \\
\hline 7.3 & $4.78 \cdot 10^{-4}$ & 0.635 & $1.31 \cdot 10^{-3}$ & $0.0500 \pm 0.0017 \pm 0.0034$ \\
\hline 7.3 & $4.78 \cdot 10^{-4}$ & 0.725 & $1.74 \cdot 10^{-3}$ & $0.0503 \pm 0.0016 \pm 0.0034$ \\
\hline 7.3 & $4.78 \cdot 10^{-4}$ & 0.815 & $2.58 \cdot 10^{-3}$ & $0.0425 \pm 0.0015 \pm 0.0021$ \\
\hline 7.3 & $4.78 \cdot 10^{-4}$ & 0.905 & $5.03 \cdot 10^{-3}$ & $0.0205 \pm 0.0010 \pm 0.0030$ \\
\hline 7.3 & $1.02 \cdot 10^{-3}$ & 0.365 & $1.60 \cdot 10^{-3}$ & $0.0531 \pm 0.0026 \pm 0.0045$ \\
\hline 7.3 & $1.02 \cdot 10^{-3}$ & 0.455 & $1.87 \cdot 10^{-3}$ & $0.0471 \pm 0.0020 \pm 0.0036$ \\
\hline 7.3 & $1.02 \cdot 10^{-3}$ & 0.545 & $2.24 \cdot 10^{-3}$ & $0.0421 \pm 0.0016 \pm 0.0031$ \\
\hline 7.3 & $1.02 \cdot 10^{-3}$ & 0.635 & $2.79 \cdot 10^{-3}$ & $0.0445 \pm 0.0015 \pm 0.0030$ \\
\hline 7.3 & $1.02 \cdot 10^{-3}$ & 0.725 & $3.71 \cdot 10^{-3}$ & $0.0413 \pm 0.0014 \pm 0.0028$ \\
\hline 7.3 & $1.02 \cdot 10^{-3}$ & 0.815 & $5.51 \cdot 10^{-3}$ & $0.0352 \pm 0.0013 \pm 0.0019$ \\
\hline 7.3 & $1.02 \cdot 10^{-3}$ & 0.905 & $1.07 \cdot 10^{-2}$ & $0.0156 \pm 0.0008 \pm 0.0023$ \\
\hline 7.3 & $2.17 \cdot 10^{-3}$ & 0.365 & $3.42 \cdot 10^{-3}$ & $0.0461 \pm 0.0025 \pm 0.0037$ \\
\hline 7.3 & $2.17 \cdot 10^{-3}$ & 0.455 & $3.99 \cdot 10^{-3}$ & $0.0385 \pm 0.0018 \pm 0.0028$ \\
\hline 7.3 & $2.17 \cdot 10^{-3}$ & 0.545 & $4.77 \cdot 10^{-3}$ & $0.0383 \pm 0.0016 \pm 0.0030$ \\
\hline 7.3 & $2.17 \cdot 10^{-3}$ & 0.635 & $5.95 \cdot 10^{-3}$ & $0.0338 \pm 0.0013 \pm 0.0021$ \\
\hline 7.3 & $2.17 \cdot 10^{-3}$ & 0.725 & $7.90 \cdot 10^{-3}$ & $0.0346 \pm 0.0013 \pm 0.0026$ \\
\hline 7.3 & $2.17 \cdot 10^{-3}$ & 0.815 & $1.17 \cdot 10^{-2}$ & $0.0293 \pm 0.0011 \pm 0.0015$ \\
\hline 7.3 & $2.17 \cdot 10^{-3}$ & 0.905 & $2.29 \cdot 10^{-2}$ & $0.0147 \pm 0.0008 \pm 0.0024$ \\
\hline 11 & $2.24 \cdot 10^{-4}$ & 0.365 & $3.53 \cdot 10^{-4}$ & $0.0866 \pm 0.0058 \pm 0.0069$ \\
\hline 11 & $2.24 \cdot 10^{-4}$ & 0.455 & $4.12 \cdot 10^{-4}$ & $0.0740 \pm 0.0042 \pm 0.0060$ \\
\hline 11 & $2.24 \cdot 10^{-4}$ & 0.545 & $4.93 \cdot 10^{-4}$ & $0.0692 \pm 0.0036 \pm 0.0049$ \\
\hline 11 & $2.24 \cdot 10^{-4}$ & 0.635 & $6.14 \cdot 10^{-4}$ & $0.0701 \pm 0.0033 \pm 0.0052$ \\
\hline 11 & $2.24 \cdot 10^{-4}$ & 0.725 & $8.16 \cdot 10^{-4}$ & $0.0702 \pm 0.0031 \pm 0.0049$ \\
\hline 11 & $2.24 \cdot 10^{-4}$ & 0.815 & $1.21 \cdot 10^{-3}$ & $0.0517 \pm 0.0025 \pm 0.0028$ \\
\hline 11 & $2.24 \cdot 10^{-4}$ & 0.905 & $2.36 \cdot 10^{-3}$ & $0.0273 \pm 0.0019 \pm 0.0046$ \\
\hline 11 & $4.78 \cdot 10^{-4}$ & 0.365 & $7.53 \cdot 10^{-4}$ & $0.0771 \pm 0.0044 \pm 0.0061$ \\
\hline 11 & $4.78 \cdot 10^{-4}$ & 0.455 & $8.77 \cdot 10^{-4}$ & $0.0652 \pm 0.0032 \pm 0.0047$ \\
\hline 11 & $4.78 \cdot 10^{-4}$ & 0.545 & $1.05 \cdot 10^{-3}$ & $0.0580 \pm 0.0026 \pm 0.0051$ \\
\hline 11 & $4.78 \cdot 10^{-4}$ & 0.635 & $1.31 \cdot 10^{-3}$ & $0.0635 \pm 0.0025 \pm 0.0046$ \\
\hline 11 & $4.78 \cdot 10^{-4}$ & 0.725 & $1.74 \cdot 10^{-3}$ & $0.0570 \pm 0.0022 \pm 0.0034$ \\
\hline 11 & $4.78 \cdot 10^{-4}$ & 0.815 & $2.58 \cdot 10^{-3}$ & $0.0483 \pm 0.0020 \pm 0.0023$ \\
\hline 11 & $4.78 \cdot 10^{-4}$ & 0.905 & $5.03 \cdot 10^{-3}$ & $0.0226 \pm 0.0014 \pm 0.0039$ \\
\hline 11 & $1.02 \cdot 10^{-3}$ & 0.365 & $1.60 \cdot 10^{-3}$ & $0.0623 \pm 0.0037 \pm 0.0052$ \\
\hline 11 & $1.02 \cdot 10^{-3}$ & 0.455 & $1.87 \cdot 10^{-3}$ & $0.0554 \pm 0.0028 \pm 0.0041$ \\
\hline 11 & $1.02 \cdot 10^{-3}$ & 0.545 & $2.24 \cdot 10^{-3}$ & $0.0513 \pm 0.0023 \pm 0.0042$ \\
\hline 11 & $1.02 \cdot 10^{-3}$ & 0.635 & $2.79 \cdot 10^{-3}$ & $0.0483 \pm 0.0020 \pm 0.0030$ \\
\hline 11 & $1.02 \cdot 10^{-3}$ & 0.725 & $3.71 \cdot 10^{-3}$ & $0.0493 \pm 0.0020 \pm 0.0035$ \\
\hline 11 & $1.02 \cdot 10^{-3}$ & 0.815 & $5.51 \cdot 10^{-3}$ & $0.0419 \pm 0.0018 \pm 0.0021$ \\
\hline 11 & $1.02 \cdot 10^{-3}$ & 0.905 & $1.07 \cdot 10^{-2}$ & $0.0182 \pm 0.0011 \pm 0.0033$ \\
\hline
\end{tabular}


Table 3 (Continued)

\begin{tabular}{|c|c|c|c|c|}
\hline$Q^{2}\left[\mathrm{GeV}^{2}\right]$ & $x$ & $x_{L}$ & $\beta$ & $F_{2}^{L N(3)}$ \\
\hline 11 & $2.17 \cdot 10^{-3}$ & 0.365 & $3.42 \cdot 10^{-3}$ & $0.0505 \pm 0.0032 \pm 0.0041$ \\
\hline 11 & $2.17 \cdot 10^{-3}$ & 0.455 & $3.99 \cdot 10^{-3}$ & $0.0447 \pm 0.0024 \pm 0.0035$ \\
\hline 11 & $2.17 \cdot 10^{-3}$ & 0.545 & $4.77 \cdot 10^{-3}$ & $0.0428 \pm 0.0021 \pm 0.0032$ \\
\hline 11 & $2.17 \cdot 10^{-3}$ & 0.635 & $5.95 \cdot 10^{-3}$ & $0.0417 \pm 0.0018 \pm 0.0034$ \\
\hline 11 & $2.17 \cdot 10^{-3}$ & 0.725 & $7.90 \cdot 10^{-3}$ & $0.0420 \pm 0.0018 \pm 0.0026$ \\
\hline 11 & $2.17 \cdot 10^{-3}$ & 0.815 & $1.17 \cdot 10^{-2}$ & $0.0339 \pm 0.0016 \pm 0.0016$ \\
\hline 11 & $2.17 \cdot 10^{-3}$ & 0.905 & $2.29 \cdot 10^{-2}$ & $0.0167 \pm 0.0011 \pm 0.0031$ \\
\hline 11 & $4.63 \cdot 10^{-3}$ & 0.365 & $7.29 \cdot 10^{-3}$ & $0.0460 \pm 0.0035 \pm 0.0041$ \\
\hline 11 & $4.63 \cdot 10^{-3}$ & 0.455 & $8.50 \cdot 10^{-3}$ & $0.0412 \pm 0.0027 \pm 0.0032$ \\
\hline 11 & $4.63 \cdot 10^{-3}$ & 0.545 & $1.02 \cdot 10^{-2}$ & $0.0348 \pm 0.0021 \pm 0.0027$ \\
\hline 11 & $4.63 \cdot 10^{-3}$ & 0.635 & $1.27 \cdot 10^{-2}$ & $0.0344 \pm 0.0019 \pm 0.0025$ \\
\hline 11 & $4.63 \cdot 10^{-3}$ & 0.725 & $1.68 \cdot 10^{-2}$ & $0.0307 \pm 0.0016 \pm 0.0018$ \\
\hline 11 & $4.63 \cdot 10^{-3}$ & 0.815 & $2.50 \cdot 10^{-2}$ & $0.0269 \pm 0.0015 \pm 0.0012$ \\
\hline 11 & $4.63 \cdot 10^{-3}$ & 0.905 & $4.87 \cdot 10^{-2}$ & $0.0157 \pm 0.0012 \pm 0.0023$ \\
\hline 16 & $4.78 \cdot 10^{-4}$ & 0.365 & $7.53 \cdot 10^{-4}$ & $0.0829 \pm 0.0059 \pm 0.0069$ \\
\hline 16 & $4.78 \cdot 10^{-4}$ & 0.455 & $8.77 \cdot 10^{-4}$ & $0.0652 \pm 0.0039 \pm 0.0049$ \\
\hline 16 & $4.78 \cdot 10^{-4}$ & 0.545 & $1.05 \cdot 10^{-3}$ & $0.0679 \pm 0.0037 \pm 0.0049$ \\
\hline 16 & $4.78 \cdot 10^{-4}$ & 0.635 & $1.31 \cdot 10^{-3}$ & $0.0666 \pm 0.0033 \pm 0.0052$ \\
\hline 16 & $4.78 \cdot 10^{-4}$ & 0.725 & $1.74 \cdot 10^{-3}$ & $0.0708 \pm 0.0034 \pm 0.0049$ \\
\hline 16 & $4.78 \cdot 10^{-4}$ & 0.815 & $2.58 \cdot 10^{-3}$ & $0.0578 \pm 0.0030 \pm 0.0030$ \\
\hline 16 & $4.78 \cdot 10^{-4}$ & 0.905 & $5.03 \cdot 10^{-3}$ & $0.0300 \pm 0.0022 \pm 0.0051$ \\
\hline 16 & $1.02 \cdot 10^{-3}$ & 0.365 & $1.60 \cdot 10^{-3}$ & $0.0787 \pm 0.0054 \pm 0.0068$ \\
\hline 16 & $1.02 \cdot 10^{-3}$ & 0.455 & $1.87 \cdot 10^{-3}$ & $0.0706 \pm 0.0043 \pm 0.0050$ \\
\hline 16 & $1.02 \cdot 10^{-3}$ & 0.545 & $2.24 \cdot 10^{-3}$ & $0.0529 \pm 0.0029 \pm 0.0040$ \\
\hline 16 & $1.02 \cdot 10^{-3}$ & 0.635 & $2.79 \cdot 10^{-3}$ & $0.0580 \pm 0.0029 \pm 0.0043$ \\
\hline 16 & $1.02 \cdot 10^{-3}$ & 0.725 & $3.71 \cdot 10^{-3}$ & $0.0568 \pm 0.0028 \pm 0.0038$ \\
\hline 16 & $1.02 \cdot 10^{-3}$ & 0.815 & $5.51 \cdot 10^{-3}$ & $0.0469 \pm 0.0024 \pm 0.0023$ \\
\hline 16 & $1.02 \cdot 10^{-3}$ & 0.905 & $1.07 \cdot 10^{-2}$ & $0.0233 \pm 0.0017 \pm 0.0041$ \\
\hline 16 & $2.17 \cdot 10^{-3}$ & 0.365 & $3.42 \cdot 10^{-3}$ & $0.0674 \pm 0.0050 \pm 0.0055$ \\
\hline 16 & $2.17 \cdot 10^{-3}$ & 0.455 & $3.99 \cdot 10^{-3}$ & $0.0546 \pm 0.0035 \pm 0.0036$ \\
\hline 16 & $2.17 \cdot 10^{-3}$ & 0.545 & $4.77 \cdot 10^{-3}$ & $0.0512 \pm 0.0030 \pm 0.0039$ \\
\hline 16 & $2.17 \cdot 10^{-3}$ & 0.635 & $5.95 \cdot 10^{-3}$ & $0.0513 \pm 0.0027 \pm 0.0042$ \\
\hline 16 & $2.17 \cdot 10^{-3}$ & 0.725 & $7.90 \cdot 10^{-3}$ & $0.0485 \pm 0.0025 \pm 0.0032$ \\
\hline 16 & $2.17 \cdot 10^{-3}$ & 0.815 & $1.17 \cdot 10^{-2}$ & $0.0416 \pm 0.0023 \pm 0.0021$ \\
\hline 16 & $2.17 \cdot 10^{-3}$ & 0.905 & $2.29 \cdot 10^{-2}$ & $0.0195 \pm 0.0015 \pm 0.0036$ \\
\hline 16 & $4.63 \cdot 10^{-3}$ & 0.365 & $7.29 \cdot 10^{-3}$ & $0.0544 \pm 0.0045 \pm 0.0039$ \\
\hline 16 & $4.63 \cdot 10^{-3}$ & 0.455 & $8.50 \cdot 10^{-3}$ & $0.0425 \pm 0.0029 \pm 0.0030$ \\
\hline 16 & $4.63 \cdot 10^{-3}$ & 0.545 & $1.02 \cdot 10^{-2}$ & $0.0417 \pm 0.0026 \pm 0.0032$ \\
\hline 16 & $4.63 \cdot 10^{-3}$ & 0.635 & $1.27 \cdot 10^{-2}$ & $0.0421 \pm 0.0024 \pm 0.0037$ \\
\hline 16 & $4.63 \cdot 10^{-3}$ & 0.725 & $1.68 \cdot 10^{-2}$ & $0.0377 \pm 0.0021 \pm 0.0021$ \\
\hline 16 & $4.63 \cdot 10^{-3}$ & 0.815 & $2.50 \cdot 10^{-2}$ & $0.0307 \pm 0.0018 \pm 0.0017$ \\
\hline 16 & $4.63 \cdot 10^{-3}$ & 0.905 & $4.87 \cdot 10^{-2}$ & $0.0151 \pm 0.0012 \pm 0.0023$ \\
\hline
\end{tabular}


Table 3 (Continued)

\begin{tabular}{|c|c|c|c|c|}
\hline$Q^{2}\left[\mathrm{GeV}^{2}\right]$ & $x$ & $x_{L}$ & $\beta$ & $F_{2}^{L N(3)}$ \\
\hline 24 & $4.78 \cdot 10^{-4}$ & 0.365 & $7.53 \cdot 10^{-4}$ & $0.0945 \pm 0.0091 \pm 0.0076$ \\
\hline 24 & $4.78 \cdot 10^{-4}$ & 0.455 & $8.77 \cdot 10^{-4}$ & $0.0903 \pm 0.0076 \pm 0.0075$ \\
\hline 24 & $4.78 \cdot 10^{-4}$ & 0.545 & $1.05 \cdot 10^{-3}$ & $0.0716 \pm 0.0054 \pm 0.0047$ \\
\hline 24 & $4.78 \cdot 10^{-4}$ & 0.635 & $1.31 \cdot 10^{-3}$ & $0.0664 \pm 0.0047 \pm 0.0049$ \\
\hline 24 & $4.78 \cdot 10^{-4}$ & 0.725 & $1.74 \cdot 10^{-3}$ & $0.0784 \pm 0.0052 \pm 0.0059$ \\
\hline 24 & $4.78 \cdot 10^{-4}$ & 0.815 & $2.58 \cdot 10^{-3}$ & $0.0640 \pm 0.0047 \pm 0.0032$ \\
\hline 24 & $4.78 \cdot 10^{-4}$ & 0.905 & $5.03 \cdot 10^{-3}$ & $0.0281 \pm 0.0029 \pm 0.0062$ \\
\hline 24 & $1.02 \cdot 10^{-3}$ & 0.365 & $1.60 \cdot 10^{-3}$ & $0.0871 \pm 0.0073 \pm 0.0070$ \\
\hline 24 & $1.02 \cdot 10^{-3}$ & 0.455 & $1.87 \cdot 10^{-3}$ & $0.0782 \pm 0.0056 \pm 0.0064$ \\
\hline 24 & $1.02 \cdot 10^{-3}$ & 0.545 & $2.24 \cdot 10^{-3}$ & $0.0679 \pm 0.0044 \pm 0.0040$ \\
\hline 24 & $1.02 \cdot 10^{-3}$ & 0.635 & $2.79 \cdot 10^{-3}$ & $0.0678 \pm 0.0040 \pm 0.0054$ \\
\hline 24 & $1.02 \cdot 10^{-3}$ & 0.725 & $3.71 \cdot 10^{-3}$ & $0.0617 \pm 0.0035 \pm 0.0044$ \\
\hline 24 & $1.02 \cdot 10^{-3}$ & 0.815 & $5.51 \cdot 10^{-3}$ & $0.0494 \pm 0.0031 \pm 0.0028$ \\
\hline 24 & $1.02 \cdot 10^{-3}$ & 0.905 & $1.07 \cdot 10^{-2}$ & $0.0231 \pm 0.0021 \pm 0.0036$ \\
\hline 24 & $2.17 \cdot 10^{-3}$ & 0.365 & $3.42 \cdot 10^{-3}$ & $0.0684 \pm 0.0060 \pm 0.0058$ \\
\hline 24 & $2.17 \cdot 10^{-3}$ & 0.455 & $3.99 \cdot 10^{-3}$ & $0.0602 \pm 0.0045 \pm 0.0046$ \\
\hline 24 & $2.17 \cdot 10^{-3}$ & 0.545 & $4.77 \cdot 10^{-3}$ & $0.0601 \pm 0.0041 \pm 0.0057$ \\
\hline 24 & $2.17 \cdot 10^{-3}$ & 0.635 & $5.95 \cdot 10^{-3}$ & $0.0545 \pm 0.0034 \pm 0.0028$ \\
\hline 24 & $2.17 \cdot 10^{-3}$ & 0.725 & $7.90 \cdot 10^{-3}$ & $0.0471 \pm 0.0029 \pm 0.0031$ \\
\hline 24 & $2.17 \cdot 10^{-3}$ & 0.815 & $1.17 \cdot 10^{-2}$ & $0.0429 \pm 0.0028 \pm 0.0020$ \\
\hline 24 & $2.17 \cdot 10^{-3}$ & 0.905 & $2.29 \cdot 10^{-2}$ & $0.0206 \pm 0.0019 \pm 0.0039$ \\
\hline 24 & $4.63 \cdot 10^{-3}$ & 0.365 & $7.29 \cdot 10^{-3}$ & $0.0531 \pm 0.0050 \pm 0.0041$ \\
\hline 24 & $4.63 \cdot 10^{-3}$ & 0.455 & $8.50 \cdot 10^{-3}$ & $0.0503 \pm 0.0040 \pm 0.0031$ \\
\hline 24 & $4.63 \cdot 10^{-3}$ & 0.545 & $1.02 \cdot 10^{-2}$ & $0.0467 \pm 0.0034 \pm 0.0038$ \\
\hline 24 & $4.63 \cdot 10^{-3}$ & 0.635 & $1.27 \cdot 10^{-2}$ & $0.0426 \pm 0.0028 \pm 0.0033$ \\
\hline 24 & $4.63 \cdot 10^{-3}$ & 0.725 & $1.68 \cdot 10^{-2}$ & $0.0421 \pm 0.0027 \pm 0.0030$ \\
\hline 24 & $4.63 \cdot 10^{-3}$ & 0.815 & $2.50 \cdot 10^{-2}$ & $0.0328 \pm 0.0022 \pm 0.0019$ \\
\hline 24 & $4.63 \cdot 10^{-3}$ & 0.905 & $4.87 \cdot 10^{-2}$ & $0.0174 \pm 0.0017 \pm 0.0028$ \\
\hline 24 & $9.87 \cdot 10^{-3}$ & 0.365 & $1.55 \cdot 10^{-2}$ & $0.0452 \pm 0.0052 \pm 0.0035$ \\
\hline 24 & $9.87 \cdot 10^{-3}$ & 0.455 & $1.81 \cdot 10^{-2}$ & $0.0421 \pm 0.0042 \pm 0.0034$ \\
\hline 24 & $9.87 \cdot 10^{-3}$ & 0.545 & $2.17 \cdot 10^{-2}$ & $0.0398 \pm 0.0035 \pm 0.0038$ \\
\hline 24 & $9.87 \cdot 10^{-3}$ & 0.635 & $2.70 \cdot 10^{-2}$ & $0.0345 \pm 0.0028 \pm 0.0018$ \\
\hline 24 & $9.87 \cdot 10^{-3}$ & 0.725 & $3.59 \cdot 10^{-2}$ & $0.0341 \pm 0.0027 \pm 0.0028$ \\
\hline 24 & $9.87 \cdot 10^{-3}$ & 0.815 & $5.34 \cdot 10^{-2}$ & $0.0266 \pm 0.0022 \pm 0.0017$ \\
\hline 24 & $9.87 \cdot 10^{-3}$ & 0.905 & $1.04 \cdot 10^{-1}$ & $0.0143 \pm 0.0015 \pm 0.0029$ \\
\hline 37 & $1.02 \cdot 10^{-3}$ & 0.365 & $1.60 \cdot 10^{-3}$ & $0.1051 \pm 0.0109 \pm 0.0088$ \\
\hline 37 & $1.02 \cdot 10^{-3}$ & 0.455 & $1.87 \cdot 10^{-3}$ & $0.0708 \pm 0.0063 \pm 0.0053$ \\
\hline 37 & $1.02 \cdot 10^{-3}$ & 0.545 & $2.24 \cdot 10^{-3}$ & $0.0726 \pm 0.0058 \pm 0.0054$ \\
\hline 37 & $1.02 \cdot 10^{-3}$ & 0.635 & $2.79 \cdot 10^{-3}$ & $0.0720 \pm 0.0052 \pm 0.0056$ \\
\hline 37 & $1.02 \cdot 10^{-3}$ & 0.725 & $3.71 \cdot 10^{-3}$ & $0.0621 \pm 0.0045 \pm 0.0036$ \\
\hline
\end{tabular}


Table 3 (Continued)

\begin{tabular}{|c|c|c|c|c|}
\hline$Q^{2}\left[\mathrm{GeV}^{2}\right]$ & $x$ & $x_{L}$ & $\beta$ & $F_{2}^{L N(3)}$ \\
\hline 37 & $1.02 \cdot 10^{-3}$ & 0.815 & $5.51 \cdot 10^{-3}$ & $0.0497 \pm 0.0039 \pm 0.0025$ \\
\hline 37 & $1.02 \cdot 10^{-3}$ & 0.905 & $1.07 \cdot 10^{-2}$ & $0.0236 \pm 0.0026 \pm 0.0044$ \\
\hline 37 & $2.17 \cdot 10^{-3}$ & 0.365 & $3.42 \cdot 10^{-3}$ & $0.0757 \pm 0.0081 \pm 0.0065$ \\
\hline 37 & $2.17 \cdot 10^{-3}$ & 0.455 & $3.99 \cdot 10^{-3}$ & $0.0667 \pm 0.0060 \pm 0.0041$ \\
\hline 37 & $2.17 \cdot 10^{-3}$ & 0.545 & $4.77 \cdot 10^{-3}$ & $0.0571 \pm 0.0046 \pm 0.0045$ \\
\hline 37 & $2.17 \cdot 10^{-3}$ & 0.635 & $5.95 \cdot 10^{-3}$ & $0.0593 \pm 0.0044 \pm 0.0042$ \\
\hline 37 & $2.17 \cdot 10^{-3}$ & 0.725 & $7.90 \cdot 10^{-3}$ & $0.0546 \pm 0.0039 \pm 0.0041$ \\
\hline 37 & $2.17 \cdot 10^{-3}$ & 0.815 & $1.17 \cdot 10^{-2}$ & $0.0440 \pm 0.0035 \pm 0.0038$ \\
\hline 37 & $2.17 \cdot 10^{-3}$ & 0.905 & $2.29 \cdot 10^{-2}$ & $0.0213 \pm 0.0024 \pm 0.0037$ \\
\hline 37 & $4.63 \cdot 10^{-3}$ & 0.365 & $7.29 \cdot 10^{-3}$ & $0.0647 \pm 0.0075 \pm 0.0053$ \\
\hline 37 & $4.63 \cdot 10^{-3}$ & 0.455 & $8.50 \cdot 10^{-3}$ & $0.0550 \pm 0.0053 \pm 0.0039$ \\
\hline 37 & $4.63 \cdot 10^{-3}$ & 0.545 & $1.02 \cdot 10^{-2}$ & $0.0493 \pm 0.0043 \pm 0.0036$ \\
\hline 37 & $4.63 \cdot 10^{-3}$ & 0.635 & $1.27 \cdot 10^{-2}$ & $0.0439 \pm 0.0035 \pm 0.0029$ \\
\hline 37 & $4.63 \cdot 10^{-3}$ & 0.725 & $1.68 \cdot 10^{-2}$ & $0.0458 \pm 0.0035 \pm 0.0027$ \\
\hline 37 & $4.63 \cdot 10^{-3}$ & 0.815 & $2.50 \cdot 10^{-2}$ & $0.0362 \pm 0.0030 \pm 0.0017$ \\
\hline 37 & $4.63 \cdot 10^{-3}$ & 0.905 & $4.87 \cdot 10^{-2}$ & $0.0177 \pm 0.0021 \pm 0.0038$ \\
\hline 37 & $9.87 \cdot 10^{-3}$ & 0.365 & $1.55 \cdot 10^{-2}$ & $0.0469 \pm 0.0057 \pm 0.0046$ \\
\hline 37 & $9.87 \cdot 10^{-3}$ & 0.455 & $1.81 \cdot 10^{-2}$ & $0.0446 \pm 0.0046 \pm 0.0030$ \\
\hline 37 & $9.87 \cdot 10^{-3}$ & 0.545 & $2.17 \cdot 10^{-2}$ & $0.0390 \pm 0.0037 \pm 0.0038$ \\
\hline 37 & $9.87 \cdot 10^{-3}$ & 0.635 & $2.70 \cdot 10^{-2}$ & $0.0372 \pm 0.0032 \pm 0.0023$ \\
\hline 37 & $9.87 \cdot 10^{-3}$ & 0.725 & $3.59 \cdot 10^{-2}$ & $0.0335 \pm 0.0028 \pm 0.0019$ \\
\hline 37 & $9.87 \cdot 10^{-3}$ & 0.815 & $5.34 \cdot 10^{-2}$ & $0.0326 \pm 0.0028 \pm 0.0018$ \\
\hline 37 & $9.87 \cdot 10^{-3}$ & 0.905 & $1.04 \cdot 10^{-1}$ & $0.0138 \pm 0.0017 \pm 0.0023$ \\
\hline 55 & $2.17 \cdot 10^{-3}$ & 0.365 & $3.42 \cdot 10^{-3}$ & $0.0962 \pm 0.0125 \pm 0.0074$ \\
\hline 55 & $2.17 \cdot 10^{-3}$ & 0.455 & $3.99 \cdot 10^{-3}$ & $0.0621 \pm 0.0072 \pm 0.0074$ \\
\hline 55 & $2.17 \cdot 10^{-3}$ & 0.545 & $4.77 \cdot 10^{-3}$ & $0.0727 \pm 0.0072 \pm 0.0045$ \\
\hline 55 & $2.17 \cdot 10^{-3}$ & 0.635 & $5.95 \cdot 10^{-3}$ & $0.0622 \pm 0.0057 \pm 0.0034$ \\
\hline 55 & $2.17 \cdot 10^{-3}$ & 0.725 & $7.90 \cdot 10^{-3}$ & $0.0540 \pm 0.0048 \pm 0.0041$ \\
\hline 55 & $2.17 \cdot 10^{-3}$ & 0.815 & $1.17 \cdot 10^{-2}$ & $0.0486 \pm 0.0048 \pm 0.0027$ \\
\hline 55 & $2.17 \cdot 10^{-3}$ & 0.905 & $2.29 \cdot 10^{-2}$ & $0.0204 \pm 0.0029 \pm 0.0039$ \\
\hline 55 & $4.63 \cdot 10^{-3}$ & 0.365 & $7.29 \cdot 10^{-3}$ & $0.0695 \pm 0.0093 \pm 0.0052$ \\
\hline 55 & $4.63 \cdot 10^{-3}$ & 0.455 & $8.50 \cdot 10^{-3}$ & $0.0614 \pm 0.0069 \pm 0.0052$ \\
\hline 55 & $4.63 \cdot 10^{-3}$ & 0.545 & $1.02 \cdot 10^{-2}$ & $0.0448 \pm 0.0048 \pm 0.0032$ \\
\hline 55 & $4.63 \cdot 10^{-3}$ & 0.635 & $1.27 \cdot 10^{-2}$ & $0.0482 \pm 0.0048 \pm 0.0035$ \\
\hline 55 & $4.63 \cdot 10^{-3}$ & 0.725 & $1.68 \cdot 10^{-2}$ & $0.0498 \pm 0.0046 \pm 0.0035$ \\
\hline 55 & $4.63 \cdot 10^{-3}$ & 0.815 & $2.50 \cdot 10^{-2}$ & $0.0415 \pm 0.0042 \pm 0.0034$ \\
\hline 55 & $4.63 \cdot 10^{-3}$ & 0.905 & $4.87 \cdot 10^{-2}$ & $0.0164 \pm 0.0023 \pm 0.0022$ \\
\hline 55 & $9.87 \cdot 10^{-3}$ & 0.365 & $1.55 \cdot 10^{-2}$ & $0.0592 \pm 0.0084 \pm 0.0048$ \\
\hline 55 & $9.87 \cdot 10^{-3}$ & 0.455 & $1.81 \cdot 10^{-2}$ & $0.0429 \pm 0.0053 \pm 0.0027$ \\
\hline
\end{tabular}


Table 3 (Continued)

\begin{tabular}{|c|c|c|c|c|}
\hline$Q^{2}\left[\mathrm{GeV}^{2}\right]$ & $x$ & $x_{L}$ & $\beta$ & $F_{2}^{L N(3)}$ \\
\hline 55 & $9.87 \cdot 10^{-3}$ & 0.545 & $2.17 \cdot 10^{-2}$ & $0.0409 \pm 0.0046 \pm 0.0030$ \\
\hline 55 & $9.87 \cdot 10^{-3}$ & 0.635 & $2.70 \cdot 10^{-2}$ & $0.0372 \pm 0.0038 \pm 0.0027$ \\
\hline 55 & $9.87 \cdot 10^{-3}$ & 0.725 & $3.59 \cdot 10^{-2}$ & $0.0338 \pm 0.0034 \pm 0.0022$ \\
\hline 55 & $9.87 \cdot 10^{-3}$ & 0.815 & $5.34 \cdot 10^{-2}$ & $0.0304 \pm 0.0032 \pm 0.0018$ \\
\hline 55 & $9.87 \cdot 10^{-3}$ & 0.905 & $1.04 \cdot 10^{-1}$ & $0.0148 \pm 0.0022 \pm 0.0037$ \\
\hline 55 & $2.10 \cdot 10^{-2}$ & 0.365 & $3.31 \cdot 10^{-2}$ & $0.0515 \pm 0.0089 \pm 0.0046$ \\
\hline 55 & $2.10 \cdot 10^{-2}$ & 0.455 & $3.86 \cdot 10^{-2}$ & $0.0347 \pm 0.0051 \pm 0.0021$ \\
\hline 55 & $2.10 \cdot 10^{-2}$ & 0.545 & $4.62 \cdot 10^{-2}$ & $0.0286 \pm 0.0038 \pm 0.0038$ \\
\hline 55 & $2.10 \cdot 10^{-2}$ & 0.635 & $5.76 \cdot 10^{-2}$ & $0.0334 \pm 0.0040 \pm 0.0018$ \\
\hline 55 & $2.10 \cdot 10^{-2}$ & 0.725 & $7.65 \cdot 10^{-2}$ & $0.0303 \pm 0.0036 \pm 0.0020$ \\
\hline 55 & $2.10 \cdot 10^{-2}$ & 0.815 & $1.14 \cdot 10^{-1}$ & $0.0266 \pm 0.0033 \pm 0.0023$ \\
\hline 55 & $2.10 \cdot 10^{-2}$ & 0.905 & $2.21 \cdot 10^{-1}$ & $0.0098 \pm 0.0018 \pm 0.0018$ \\
\hline 82 & $4.63 \cdot 10^{-3}$ & 0.365 & $7.29 \cdot 10^{-3}$ & $0.0588 \pm 0.0099 \pm 0.0045$ \\
\hline 82 & $4.63 \cdot 10^{-3}$ & 0.455 & $8.50 \cdot 10^{-3}$ & $0.0537 \pm 0.0080 \pm 0.0036$ \\
\hline 82 & $4.63 \cdot 10^{-3}$ & 0.545 & $1.02 \cdot 10^{-2}$ & $0.0465 \pm 0.0061 \pm 0.0036$ \\
\hline 82 & $4.63 \cdot 10^{-3}$ & 0.635 & $1.27 \cdot 10^{-2}$ & $0.0520 \pm 0.0063 \pm 0.0052$ \\
\hline 82 & $4.63 \cdot 10^{-3}$ & 0.725 & $1.68 \cdot 10^{-2}$ & $0.0480 \pm 0.0056 \pm 0.0033$ \\
\hline 82 & $4.63 \cdot 10^{-3}$ & 0.815 & $2.50 \cdot 10^{-2}$ & $0.0417 \pm 0.0053 \pm 0.0040$ \\
\hline 82 & $4.63 \cdot 10^{-3}$ & 0.905 & $4.87 \cdot 10^{-2}$ & $0.0180 \pm 0.0032 \pm 0.0028$ \\
\hline 82 & $9.87 \cdot 10^{-3}$ & 0.365 & $1.55 \cdot 10^{-2}$ & $0.0484 \pm 0.0085 \pm 0.0041$ \\
\hline 82 & $9.87 \cdot 10^{-3}$ & 0.455 & $1.81 \cdot 10^{-2}$ & $0.0514 \pm 0.0077 \pm 0.0036$ \\
\hline 82 & $9.87 \cdot 10^{-3}$ & 0.545 & $2.17 \cdot 10^{-2}$ & $0.0366 \pm 0.0052 \pm 0.0025$ \\
\hline 82 & $9.87 \cdot 10^{-3}$ & 0.635 & $2.70 \cdot 10^{-2}$ & $0.0507 \pm 0.0064 \pm 0.0038$ \\
\hline 82 & $9.87 \cdot 10^{-3}$ & 0.725 & $3.59 \cdot 10^{-2}$ & $0.0361 \pm 0.0044 \pm 0.0027$ \\
\hline 82 & $9.87 \cdot 10^{-3}$ & 0.815 & $5.34 \cdot 10^{-2}$ & $0.0311 \pm 0.0041 \pm 0.0029$ \\
\hline 82 & $9.87 \cdot 10^{-3}$ & 0.905 & $1.04 \cdot 10^{-1}$ & $0.0170 \pm 0.0030 \pm 0.0027$ \\
\hline 82 & $2.10 \cdot 10^{-2}$ & 0.365 & $3.31 \cdot 10^{-2}$ & $0.0477 \pm 0.0093 \pm 0.0040$ \\
\hline 82 & $2.10 \cdot 10^{-2}$ & 0.455 & $3.86 \cdot 10^{-2}$ & $0.0319 \pm 0.0052 \pm 0.0025$ \\
\hline 82 & $2.10 \cdot 10^{-2}$ & 0.545 & $4.62 \cdot 10^{-2}$ & $0.0366 \pm 0.0053 \pm 0.0024$ \\
\hline 82 & $2.10 \cdot 10^{-2}$ & 0.635 & $5.76 \cdot 10^{-2}$ & $0.0372 \pm 0.0050 \pm 0.0030$ \\
\hline 82 & $2.10 \cdot 10^{-2}$ & 0.725 & $7.65 \cdot 10^{-2}$ & $0.0443 \pm 0.0057 \pm 0.0028$ \\
\hline 82 & $2.10 \cdot 10^{-2}$ & 0.815 & $1.14 \cdot 10^{-1}$ & $0.0241 \pm 0.0035 \pm 0.0020$ \\
\hline 82 & $2.10 \cdot 10^{-2}$ & 0.905 & $2.21 \cdot 10^{-1}$ & $0.0132 \pm 0.0026 \pm 0.0027$ \\
\hline
\end{tabular}

obtained values of the parameters $a=0.052 \pm 0.003$ and $\Lambda=0.416 \pm 0.052 \mathrm{GeV}$ are in reasonable agreement with those obtained in the analysis of the proton structure function $F_{2}$ [47]. This demonstrates the similarity between the $Q^{2}$ evolution of $F_{2}^{L N(3)}$ and the $Q^{2}$ evolution of the proton structure function $F_{2}$.

Since pion exchange dominates leading neutron production at high $x_{L}$ and low $p_{T}$, the measurement of $F_{2}^{L N(3)}$ in the range $0.68<x_{L}<0.77$ can be used to estimate the pion structure function at low Bjorken- $x$, following the pro- cedure introduced in [1]. Assuming proton vertex factorisation, which is supported by the present data as explained above, the quantity $F_{2}^{L N(3)} / \Gamma_{\pi}$ can be interpreted as being equal to the structure function of the pion, where

$\Gamma_{\pi}\left(x_{L}\right)=\int_{t_{0}}^{t_{\min }} f_{\pi / p}\left(x_{L}, t\right) \mathrm{d} t$

is the integral of the pion flux over the measured $t$-range, where $t_{0}$ and $t_{\min }$ are given by (5). The pion flux from (7) used for the RAPGAP- $\pi$ simulation yields $\Gamma_{\pi}=0.13$ for 


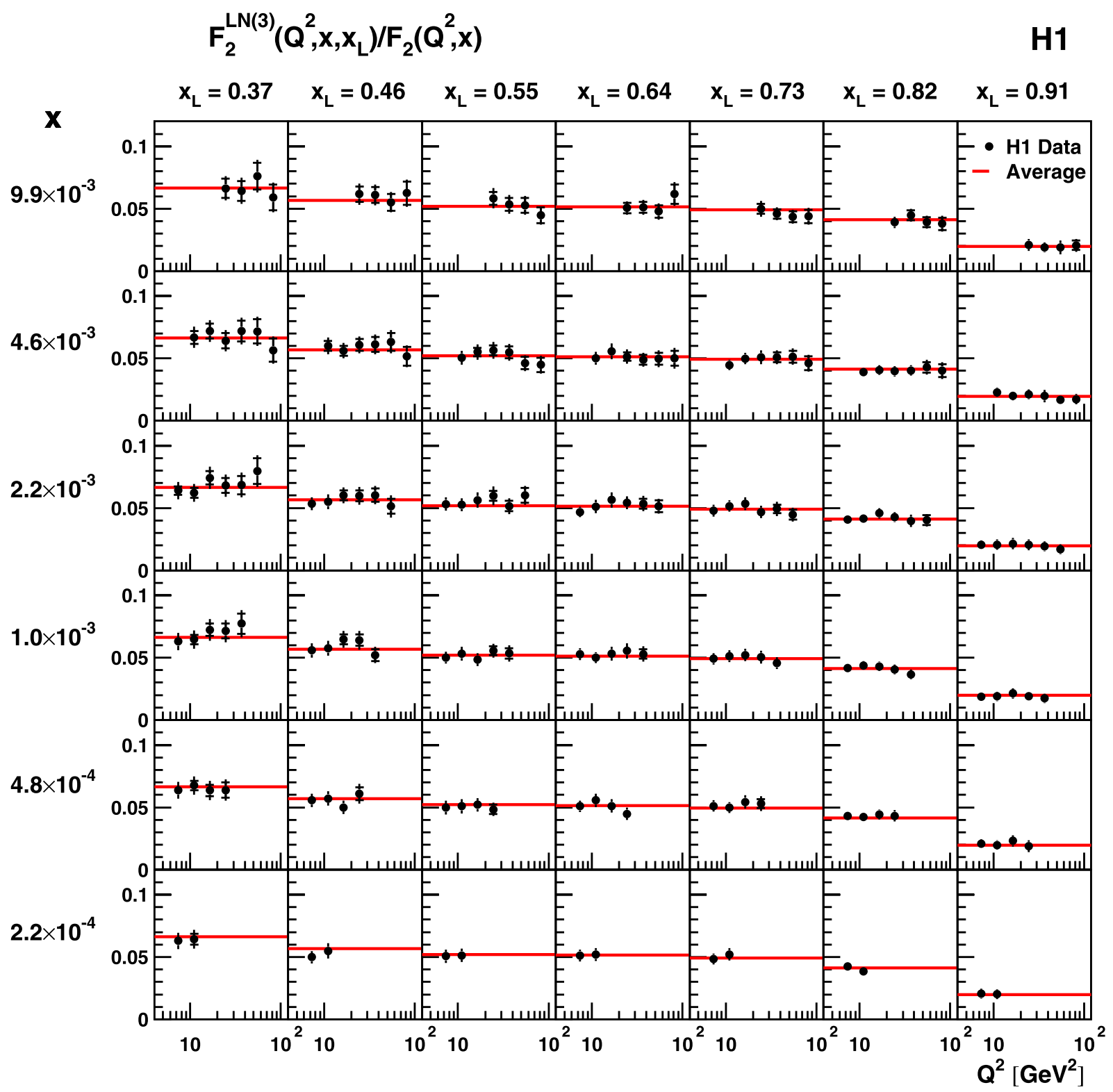

Fig. 6 The ratio of the semi-inclusive structure function $F_{2}^{L N(3)}\left(Q^{2}, x, x_{L}\right)$, for neutrons with $p_{T}<0.2 \mathrm{GeV}$, to the proton structure function $F_{2}\left(Q^{2}, x\right)$ obtained from the H1PDF2009 fit to inclusive DIS data [32]. The lines show the average value for a given $x_{L}$ bin

$p_{T}^{\max }=0.2 \mathrm{GeV}$ at $x_{L}=0.73$, which is the central value of the chosen $x_{L}$ range. Using other parameterisations of the pion flux, e.g. from $[9,11-13]$, leads to values of the pion flux integral which may differ by up to $30 \%$. In this evaluation of the pion structure function, contributions from background processes like the exchange of $\rho$ and $a_{2}$-mesons, proton diffractive dissociation and $\Delta$ production are not taken into account. Within the narrow $x_{L}$ range considered here they are only expected to affect the absolute normalisation of the results. The contribution of neutrons from fragmentation is of order $25-35 \%$, as estimated using the DJANGO simulation. The relative size of this contribution is largely independent of $Q^{2}$ and $\beta$ and thus has little impact on the shape of the distribution.

Figure 8 shows $F_{2}^{L N(3)} / \Gamma_{\pi}$ as a function of $Q^{2}$ in bins of $\beta$ while Fig. 9 shows $F_{2}^{L N(3)} / \Gamma_{\pi}$ as a function of $\beta$ in bins of $Q^{2}$. In Fig. 9 the contribution of neutrons from fragmentation, as predicted by DJANGO and scaled by a weighting factor 1.2, as described in Sect. 3.4, is indicated. The data are compared to predictions of the parameterisations of the pion structure function GRSc- $\pi$ [48] and ABFKW- $\pi$ [49]. The measurements are also compared to the H1PDF2009 parameterisation of the proton structure function $F_{2}\left(Q^{2}, x\right)$ [32] which is scaled by a factor of $2 / 3$ in order to naively account for the different number of valence quarks in the pion 


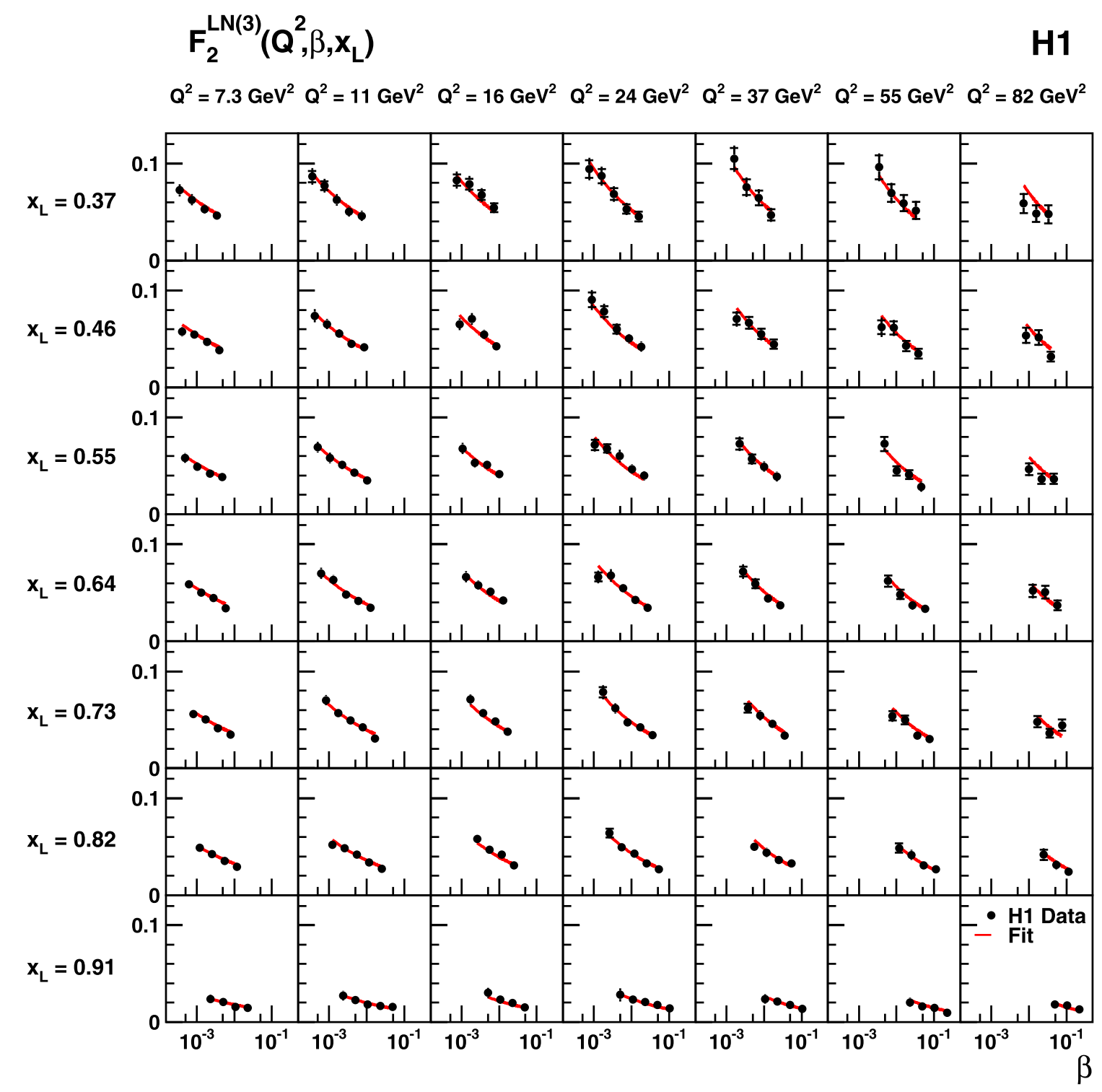

Fig. 7 The semi-inclusive structure function $F_{2}^{L N(3)}\left(Q^{2}, \beta, x_{L}\right)$, for neutrons with $p_{T}<0.2 \mathrm{GeV}$, shown as a function of $\beta$ in bins of $Q^{2}$ and $x_{L}$. The lines are the results of the fit with a function $c\left(x_{L}\right) \cdot \beta^{-\lambda\left(Q^{2}\right)}$ as described in Sect. 4

and proton, respectively. The values of $F_{2}\left(Q^{2}, x\right)$ are calculated at Bjorken- $x$ equal to $\beta$. The $Q^{2}$ distribution exhibits a rise with increasing $Q^{2}$ (i.e. scaling violation) for all $\beta$ values in the measured range, which is similar in size and shape to that seen in the parameterisations of the inclusive structure functions of both the pion and proton (Fig. 8). The $\beta$ distributions show a steep rise with decreasing $\beta$ for all $Q^{2}$ values (Fig. 9). This behaviour is in reasonable agreement with the pion and proton structure function parameterisations. In absolute values the parameterisations lie above the measurements. Other parameterisations of the pion structure function $[50,51]$ were ruled out by previous $\mathrm{H} 1$ and ZEUS measurements $[1,2]$ as they show a much flatter behaviour as a function of $\beta$.
The comparison of the measured $F_{2}^{L N(3)} / \Gamma_{\pi}$ and the parameterisations of the pion structure function is affected by uncertainties on the pion flux normalisation, as explained above. It may also depend on absorptive corrections [52-55], which are not taken into account in this analysis. Neutron absorption may occur through rescattering which transforms the neutron into a charged baryon or shifts the neutron to lower energy or higher $p_{T}$.

The results presented here are consistent with the previous measurement by the $\mathrm{H} 1$ Collaboration [1]. A similar analysis has been published by the ZEUS Collaboration [2]. There is good agreement between the two cross section measurements. For the extraction of the pion structure function, the flux factor normalisations used in the ZEUS analysis are 


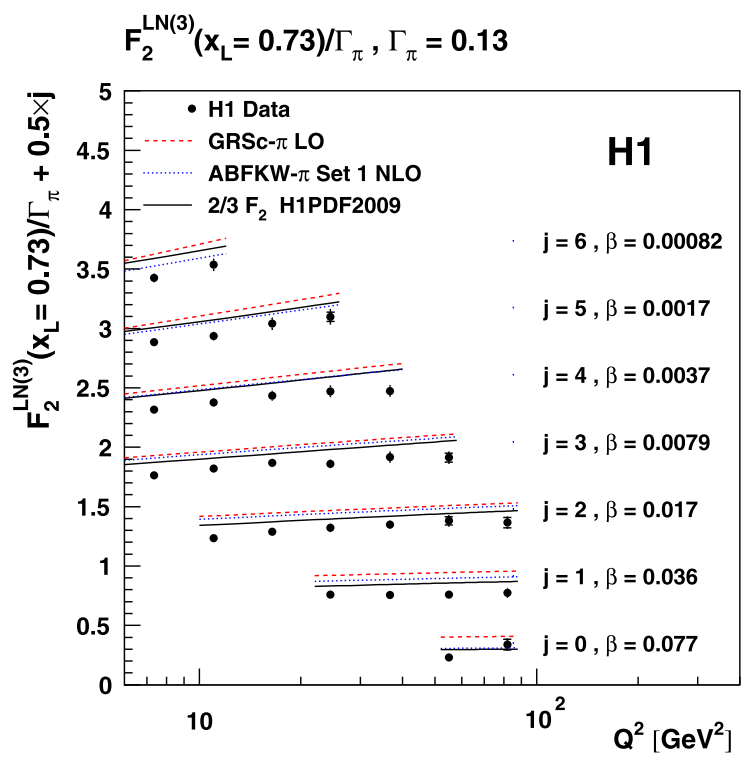

Fig. 8 The semi-inclusive structure function $F_{2}^{L N(3)}$, for neutrons with $p_{T}<0.2 \mathrm{GeV}$, divided by the pion flux $\Gamma_{\pi}$ integrated over $t$ at the central value $x_{L}=0.73$, shown as a function of $Q^{2}$ in bins of $\beta$. The pion flux is defined in (7). The data are compared to two different parameterisations of the pion structure function $F_{2}^{\pi}[48,49]$ and to the H1PDF2009 parameterisation of the proton structure function [32], which has been scaled by $2 / 3$ different from the one used here. Within the normalisation uncertainties, the $\mathrm{H} 1$ and ZEUS results agree.

\section{Summary}

The cross section for leading neutron production in deepinelastic positron-proton scattering $d \sigma / d x_{L}$ and the semiinclusive structure function $F_{2}^{L N(3)}\left(Q^{2}, x, x_{L}\right)$ are measured in the kinematic region $6<Q^{2}<100 \mathrm{GeV}^{2}$, $1.5 \cdot 10^{-4}<x<3 \cdot 10^{-2}, 0.32<x_{L}<0.95$ and $p_{T}<$ $0.2 \mathrm{GeV}$. The present measurements have experimental uncertainties of 10 to $15 \%$.

The measurements are well described by a Monte Carlo simulation including neutron production in fragmentation and neutrons produced from $\pi^{+}$exchange, as predicted by the DJANGO and RAPGAP programs respectively. At large $x_{L} \gtrsim 0.7$ the $\pi^{+}$-exchange process dominates.

Within the measured kinematic range, the semi-inclusive structure function $F_{2}^{L N(3)}$ and the inclusive structure function $F_{2}$ have similar $\left(Q^{2}, x\right)$ behaviour, which is consistent with the hypothesis of limiting fragmentation. The dependence of $F_{2}^{L N(3)}$ on the variable $\beta$ is similar for all $x_{L}$ bins, in accordance with the expectation from proton vertex factorisation. The scaling violations observed in $F_{2}^{L N(3)}$ are
Fig. 9 The semi-inclusive structure function $F_{2}^{L N(3)}$, for neutrons with $p_{T}<0.2 \mathrm{GeV}$, divided by the pion flux $\Gamma_{\pi}$ integrated over $t$ at the central value $x_{L}=0.73$, shown as a function of $\beta$ in bins of $Q^{2}$. The pion flux is defined in (7). The data are compared to two different parameterisations of the pion structure function $F_{2}^{\pi}[48,49]$ and to the H1PDF2009 parameterisation of the proton structure function [32], which has been scaled by $2 / 3$. The contribution of neutrons from fragmentation, as predicted by DJANGO and scaled by a factor 1.2 , as described in Sect. 3.4, is indicated

$$
F_{2}^{\mathrm{LN}(3)}\left(x_{\mathrm{L}}=0.73\right) / \Gamma_{\pi}, \Gamma_{\pi}=0.13
$$

H1
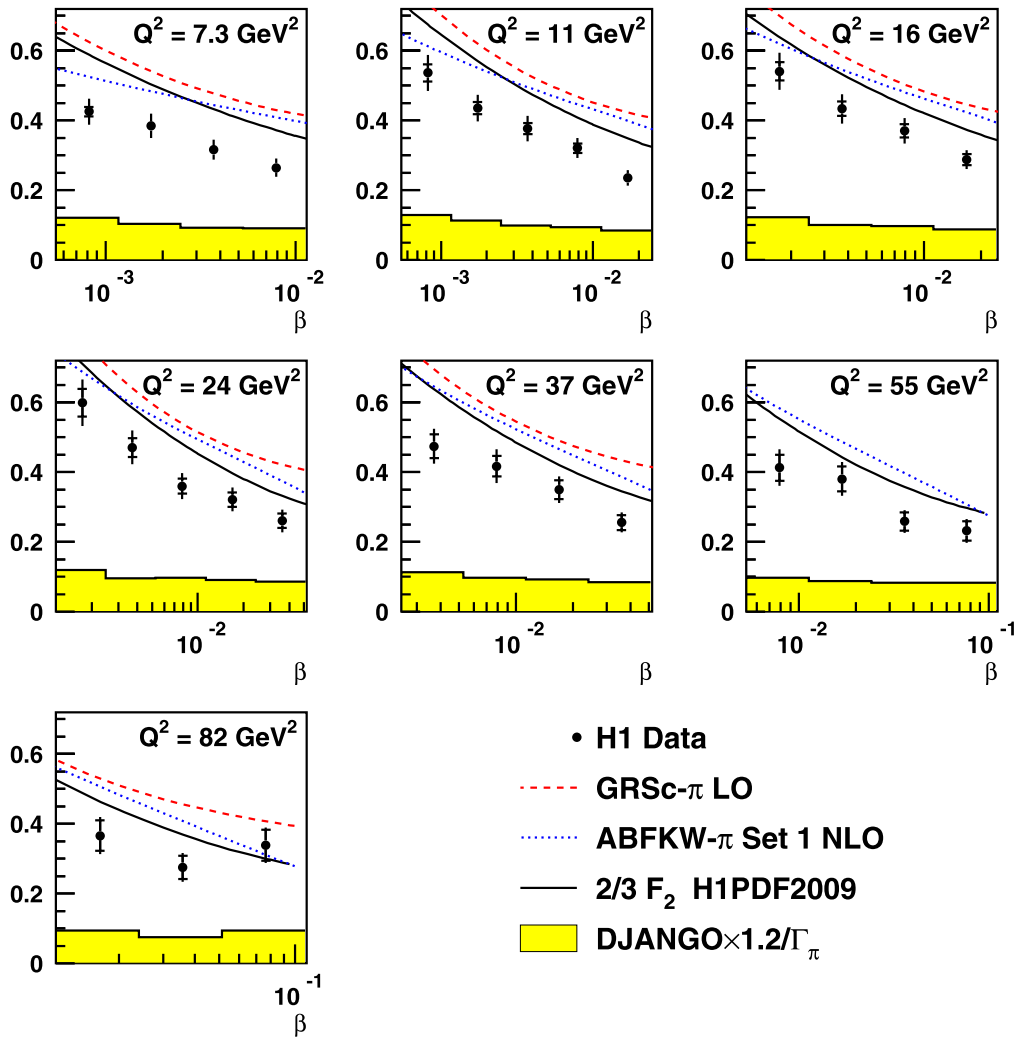

- H1 Data

GRSc- $\pi$ LO

ABFKW- $\pi$ Set 1 NLO

2/3 F H1PDF2009

DJANGO $\times 1.2 / \Gamma_{\pi}$ 
similar in size and shape to those seen in the parameterisations of the inclusive structure functions of the pion and the proton. The data are used to estimate the structure function of the pion, up to uncertainties on the background contribution and the overall normalisation, in the framework of the one pion exchange model for the neutron kinematic range $0.68<x_{L}<0.77$ and $p_{T}<0.2 \mathrm{GeV}$.

Acknowledgements We are grateful to the HERA machine group whose outstanding efforts have made this experiment possible. We thank the engineers and technicians for their work in constructing and maintaining the $\mathrm{H} 1$ detector, our funding agencies for financial support, the DESY technical staff for continual assistance and the DESY directorate for support and for the hospitality which they extend to the non-DESY members of the collaboration.

Open Access This article is distributed under the terms of the Creative Commons Attribution Noncommercial License which permits any noncommercial use, distribution, and reproduction in any medium, provided the original author(s) and source are credited.

\section{References}

1. C. Adloff et al. (H1 Collaboration), Eur. Phys. J. C 6, 587 (1999). hep-ex/9811013

2. S. Chekanov et al. (ZEUS Collaboration), Nucl. Phys. B 637, 3 (2002). hep-ex/0205076

3. J. Breitweg et al. (ZEUS Collaboration), Nucl. Phys. B 596, 3 (2001). hep-ex/0010019

4. S. Chekanov et al. (ZEUS Collaboration), Phys. Lett. B 610, 199 (2005). hep-ex/0404002

5. A. Aktas et al. (H1 Collaboration), Eur. Phys. J. C 41, 273 (2005). hep-ex/0501074

6. S. Chekanov et al. (ZEUS Collaboration), Phys. Lett. B 590, 143 (2004). hep-ex/0401017

7. S. Chekanov et al. (ZEUS Collaboration), Nucl. Phys. B 776, 1 (2007). hep-ex/0702028

8. J.D. Sullivan, Phys. Rev. D 5, 1732 (1972)

9. M. Bishari, Phys. Lett. B 38, 510 (1972)

10. H. Holtmann et al., Phys. Lett. B 338, 363 (1994)

11. B. Kopeliovich, B. Povh, I. Potashnikova, Z. Phys. C 73, 125 (1996). hep-ph/9601291

12. M. Przybycien, A. Szczurek, G. Ingelman, Z. Phys. C 74, 509 (1997). hep-ph/9606294

13. A. Szczurek, N.N. Nikolaev, J. Speth, Phys. Lett. B 428, 383 (1998). hep-ph/9712261

14. V.A. Khoze, A.D. Martin, M.G. Ryskin, Eur. Phys. J. C 48, 797 (2006). hep-ph/0606213

15. E. Anassontzis et al., Phys. Rev. D 38, 1377 (1988)

16. J.S. Conway et al., Phys. Rev. D 39, 92 (1989)

17. J. Badier et al. (NA3 Collaboration), Z. Phys. C 18, 281 (1983)

18. B. Betev et al. (NA10 Collaboration), Z. Phys. C 28, 9 (1985)

19. C. De Marzo et al. (NA24 Collaboration), Phys. Rev. D 36, 8 (1987)

20. M. Bonesini et al. (WA70 Collaboration), Z. Phys. C 37, 535 (1988)

21. J. Benecke et al., Phys. Rev. 188, 2159 (1969)
22. T.T. Chou, C.-N. Yang, Phys. Rev. D 50, 590 (1994)

23. C. Adloff et al. (H1 Collaboration), Z. Phys. C 76, 613 (1997). hep-ex/9708016

24. I. Abt et al. (H1 Collaboration), Nucl. Instrum. Methods A 386 310 (1997)

25. I. Abt et al. (H1 Collaboration), Nucl. Instrum. Methods A $\mathbf{3 8 6}$, 348 (1997)

26. R.D. Appuhn et al. (H1 SPACAL Group), Nucl. Instrum. Methods A 386, 397 (1997)

27. D. Pitzl et al., Nucl. Instrum. Methods A 454, 334 (2000). hep-ex/0002044

28. B. Andrieu et al. (H1 Calorimeter Group), Nucl. Instrum. Methods A 336, 460 (1993)

29. C. Kleinwort, H1 alignment experience, in Proceedings of the First LHC Detector Alignment Workshop, ed. by S. Blusk et al., CERN-2007-004, p. 41

30. B. Andrieu et al. (H1 Calorimeter Group), Nucl. Instrum. Methods A 336, 499 (1993)

31. B. Andrieu et al. (H1 Calorimeter Group), Nucl. Instrum. Methods A 350, 57 (1994)

32. F.D. Aaron et al. (H1 Collaboration), Eur. Phys. J. C 64, 561 (2009). doi:10.1140/epjc/s10052-009-1169-x

33. R. Brun et al., GEANT3, CERN-DD/EE/84-1

34. K. Charchula, G.A. Schuler, H. Spiesberger, DJANGOH 1.4. Comput. Phys. Commun. 81, 381 (1994)

35. L. Lönnblad, ARIADNE 4.10. Comput. Phys. Commun. 71, 15 (1992)

36. B. Andersson et al., JETSET 7.41. Phys. Rept. 97, 31 (1983)

37. H. Jung, RAPGAP 3.1. Comput. Phys. Commun. 86, 147 (1995)

38. A. Kwiatkowski, H. Spiesberger, H.J. Möhring, Comput. Phys. Commun. 69, 155 (1992)

39. R.G.E. Timmermans, T.A. Rijken, J.J. de Swart, Phys. Rev. Lett. 67, $1074(1991)$

40. M. Glück, E. Reya, A. Vogt, Z. Phys. C 53, 127 (1992)

41. M. Glück, E. Reya, A. Vogt, Z. Phys. C 53, 651 (1992)

42. R. Engel, J. Ranft, PHOJET 1.0. Phys.Rev. D 54, 4244 (1996). hep-ph/9509373

43. B. List, A. Mastroberardino, DIFFVM: a Monte Carlo generator for diffractive processes in ep scattering, in Proceedings of the Workshop "Monte Carlo Generators for HERA Physics", ed. by A.T. Doyle et al., DESY-PROC-1999-02, p. 396

44. A. Edin, G. Ingelman, J. Rathsman, Phys. Lett. B 366, 371 (1996). hep-ph/9508386

45. G. Ingelman, A. Edin, J. Rathsman, Comput. Phys. Commun. 101, 108 (1997). hep-ph/9605286

46. J. Rathsman, Phys. Lett. B 452, 364 (1999). hep-ph/9812423

47. C. Adloff et al. (H1 Collaboration), Phys. Lett. B 520, 183 (2001). hep-ex/0108035

48. M. Glück, E. Reya, I. Schienbein, Eur. Phys. J. C 10, 313 (1999). hep-ph/9903288

49. P. Aurenche et al., Phys. Lett. B 233, 517 (1989)

50. J.F. Owens, Phys. Rev. D 30, 943 (1984)

51. P.J. Sutton et al., Phys. Rev. D 45, 2349 (1992)

52. N.N. Nikolaev, J. Speth, B.G. Zakharov, hep-ph/9708290

53. U. D’Alesio, H.J. Pirner, Eur. Phys. J. A 7, 109 (2000). hep-ph/9806321

54. A.B. Kaidalov et al., Eur. Phys. J. C 47, 385 (2006). hep-ph/0602215

55. B. Kopeliovich et al., Phys. Rev. D 78, 014031 (2008). arXiv:0805.4534 